\title{
The cost-effectiveness of different approaches to exercise and corticosteroid injection for Subacromial pain (impingement) syndrome
}

\begin{abstract}
Authors:
Raymond Oppong ${ }^{1,4}$, Sue Jowett ${ }^{1,4}$, Martyn Lewis ${ }^{1,3}$, Edward Roddy ${ }^{1,2}$, Reuben O Ogollah ${ }^{1,3}$, Irena Zwierska $^{1,3}$, Praveen Datta ${ }^{5}$, Alison Hall ${ }^{1}$, Elaine Hay ${ }^{1,2}$, Julie Shufflebotham ${ }^{1,6}$, Kay Stevenson ${ }^{1,2}$, Danielle A van der Windt ${ }^{1}$, Julie Young ${ }^{1,3}$, Nadine E Foster ${ }^{1,3}$.
\end{abstract}

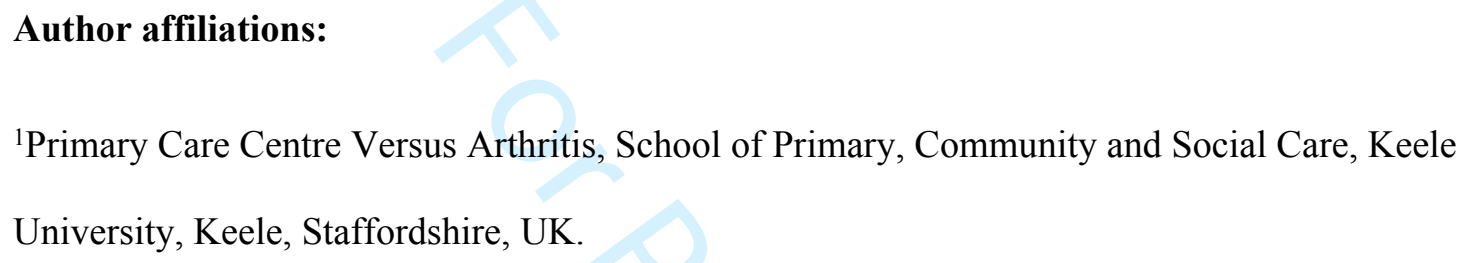

${ }^{6}$ Midlands Partnership NHS Foundation Trust, Newcastle-under-Lyme, Staffordshire, UK

\section{Corresponding author:}

Dr Sue Jowett, Health Economics Unit, Institute of Applied Health Research, University of Birmingham, B15 2TT

e-mail: s.jowett@bham.ac.uk

ORCID iD: 0000-0001-8936-3745 


\begin{abstract}
:
Objectives: To determine whether physiotherapist-led exercise intervention and ultrasoundguided subacromial corticosteroid injection is cost-effective when compared to standard advice and exercise leaflet and unguided injection in patients with subacromial pain (impingement) syndrome.
\end{abstract}

Methods: An incremental cost-utility analysis using patient responses to the five-level EuroQoL-5D (EQ-5D-5L) questionnaire was undertaken from a health care perspective alongside a 2x2 factorial randomised trial with 256 participants over a 12-month follow-up period. Uncertainty was explored through the use of cost-effectiveness acceptability curves.

Results: The cost-utility analysis indicated that physiotherapist-led exercise was associated with an incremental cost of $£ 155.99$ (95\% CI: 69.02 to 241.93 ) and 0.031 (95\% CI: -0.01 to 0.07) additional quality-adjusted life-years (QALYs), an incremental cost-effectiveness ratio (ICER) of $£ 5,031$ per QALY gained and an $85 \%$ chance of being cost-effective at a threshold of $£ 20,000$ per QALY gained compared to the advice and exercise leaflet. Ultrasound-guided injection was associated with an incremental cost of $£ 15.89$ (95\% CI: -59.36 to 109.86$)$ and 0.024 (95\% CI: -0.02 to 0.07$)$ additional QALYs, an ICER of $£ 662$ per QALY gained and a $83 \%$ chance of being cost-effective at a threshold of $£ 20,000$ per QALY gained compared to unguided injection.

Conclusion: Physiotherapist-led exercise was cost-effective compared to the advice and exercise leaflet, and ultrasound-guided injection was cost-effective when compared to unguided injection.

Clinical trial registration number: ISRCTN (42399123)

Keywords: Cost-effectiveness, Subacromial pain (impingement) syndrome , Exercise, Corticosteroid injection, EQ-5D 
Rheumatology key messages:

- Guidelines recommend non-surgical options for the management of Subacromial pain (impingement) syndrome-.

- Physiotherapist-led exercise and ultrasound-guided corticosteroid injection appear to increase costs but lead to improved quality of life compared to unguided injection and advice and exercise leaflet.

- Physiotherapist-led exercise and ultrasound-guided injection are likely to be costeffective. 


\section{Introduction}

Subacromial pain (impingement) syndrome (SAPS) is the most common cause of shoulder pain. The prevalence of SAPS increases with age and places a substantial burden on health systems, with estimates from GP consultations suggesting that approximately $16 \%$ of adults reporting musculoskeletal pain had shoulder pain [1-2]. Shoulder pain poses a considerable burden to society, accounting for a substantial number of days off work and associated productivity costs [3-4]. Treatment guidelines recommend initial non-surgical management including education/self-management advice, exercise, corticosteroid injection, and analgesia [5-6].

In order to explore how to optimise outcomes from exercise and subacromial corticosteroid injection, a randomised controlled trial was conducted to assess whether better improvements in pain and function were obtained with (1) a physiotherapist-led, individualised, supervised and progressed exercise programme versus a standardised advice and exercise leaflet, and (2) ultrasound-guided subacromial corticosteroid injection versus unguided injection. The clinical results of the trial showed that physiotherapist-led exercise was more effective than an exercise leaflet whilst ultrasound-guidance conferred no additional benefit over unguided corticosteroid injection [7]. In addition to establishing the effectiveness of interventions for the management of shoulder pain, it is also important to determine whether these interventions offer value for money. Previous studies assessing the cost-effectiveness of interventions for the treatment of SAPS have focused on a comparison of corticosteroid injection and exercise [2] and have not compared different methods of guiding corticosteroid injection, nor the manner in which the exercise intervention was delivered.

The primary aim of this economic analysis was to determine whether a physiotherapist-led exercise intervention is cost-effective compared to a standard advice and exercise leaflet, and 
whether ultrasound-guided subacromial corticosteroid injection is cost-effective when compared to unguided injection.

\section{Methods}

A within-trial economic evaluation was conducted alongside a $2 \times 2$ factorial randomised trial in patients with SAPS [7]. Using block randomisation, participants who met the inclusion criteria were randomly assigned on a 1:1:1:1 basis to one of four interventions:

1. Ultrasound-guided subacromial corticosteroid injection and physiotherapist-led individualised, supervised and progressed exercise.

2. Ultrasound-guided subacromial corticosteroid injection and an advice and exercise leaflet.

3. Unguided subacromial corticosteroid injection and physiotherapist-led individualised, supervised and progressed exercise

4. Unguided subacromial corticosteroid injection and an advice and exercise leaflet.

The main outcome for the economic analysis was the quality-adjusted life-year (QALY), over a 12-month follow-up period. The base case analysis was carried out from a health care perspective including health care costs incurred by the NHS and patients. To be consistent with the clinical study, the 'at the margins' approach which assumes that interventions are independent was adopted for the base case scenario [8-9]. The trial, including this economic analysis, was approved by The Black Country Research Ethics Committee (10/H1202/72). Trial registration number (ISRCTN42399123). 


\section{Data collection}

\section{Resource use and costs}

Data on health care resource use were obtained from participant responses to self-report questionnaires administered at 6 weeks, 6 months and 12 months. Resource use data concentrated on visits to health care professionals in primary and secondary care, medical investigations/interventions, and both prescribed and over the counter medications. Data obtained from questionnaire responses were aggregated to generate overall resource use over the 12-month follow-up period. The questionnaires also requested information on time off work over 12 months which was used to estimate productivity losses.

Unit cost data were obtained from the following sources: British National Formulary, NHS reference costs and the PSSRU publication on unit cost of health and social care [10-12] (Supplementary Table 1). 2012/2013 costs were inflated to 2019 prices using the consumer price index.

\section{Trial intervention cost}

Information on the intervention costs was obtained from trial data and all resources required to deliver the interventions were determined.

Physiotherapist-led exercise versus advice and leaflet: For the physiotherapist visits, the unit cost associated with a contact with the physiotherapist was multiplied by the number of physiotherapy contacts. Information on the number of physiotherapist contacts was obtained from case report forms that physiotherapists completed. For the advice and exercise leaflet, only the cost associated with the leaflet was included since all participants had the index consultation where the injection was performed on all patients. Full details of the intervention can be found elsewhere [13]. 
Ultrasound versus unguided injection: It was assumed that since all patients had a similar injection, the only difference between the ultrasound and unguided injection was the cost of the ultrasound. The cost of the ultrasound machine was estimated to be $£ 35,000$, and in order to work out a cost per patient, it was assumed that the life expectancy of the machine is 6 years and that the machine is used about 1872 times a year (this value was obtained through consultation with experts). Applying the annuity factor at an interest rate of 3\%, the cost of the ultrasound machine per patient was $£ 3.45$.

\section{Health Outcomes}

Using participant responses to the 5-level version of the EQ-5D-5L questionnaire [14], quality of life was measured at baseline, 6 weeks, 6 months and 12 months. EQ-5D-5L was mapped back to the EQ-5D 3L valuation set as currently recommended by NICE [15] and used to generate the EQ-5D index scores which were then used to derive QALYs. The mapping algorithm developed by Van Hout et al. 2012 was used for this purpose [16].

\section{Statistical Analysis}

The economic analysis was carried out on an intention to treat basis, with the aim to estimate the difference in costs and QALYs between the physiotherapist-led exercise intervention and the advice and exercise leaflet and between the ultrasound-guided and unguided subacromial corticosteroid injections. Over the 12-month period, missing EQ-5D scores and costs at one or more of the time points were accounted for using multiple imputation methodology to impute 25 separate datasets [17]. The number of datasets were chosen in order to adequately account for the level of missing data in the study. The approach adopted in this study used chained equations with predictive mean matching in order to ensure that the missing values are aligned to the nearest best-fitting values in the dataset [18]. This approach is particularly useful for cost and EQ-5D data which have skewed distributions, and ensures that imputed values do not go 
out of the plausible range. The coefficients were then pooled across the multiply imputed datasets using the Rubin's rule to obtain single estimates of the corresponding population parameters [17].

The imputation model was fitted with gender, age, smoking status at baseline, baseline SPADI score and health status at baseline as predictors. Using the area under the curve approach [19], QALYs over the 12-month period were estimated for each participant. Total health care costs over the 12-month period were calculated by multiplying the resource items by the respective unit cost and summing over all resource use items. Differences in mean costs and QALYs between trial arms were estimated and QALYs were adjusted to control for imbalances in baseline utility between the interventions [20].

Incremental cost effectiveness ratios (ICERs) were estimated by dividing the difference in mean cost between two interventions (physiotherapist-led exercise versus advice and exercise leaflet and ultrasound-guided versus unguided injection) by the difference in mean QALYs. Non-parametric bootstrapping was used to illustrate and quantify uncertainty. To determine the probability of an intervention being deemed cost-effective compared to an alternative intervention (physiotherapist-led exercise versus advice and exercise leaflet and ultrasoundguided versus unguided injection), cost-effectiveness acceptability curves (CEACs) were constructed [21]. This shows the probability that an intervention is cost-effective, relative to the chosen comparator, across a range of values that represent a decision makers' willingness to pay for an additional QALY. All the analyses were carried out in STATA version 12 [22].

Costs for productivity losses were computed through the human capital approach using data collected on time off work and employment. Productivity losses were obtained by the product of lost productivity time and the mean hourly wage of patients classified from data on wages 
corresponding to self-reported baseline occupation (derived from the Annual Surveys of Health Evaluation by the Office of National Statistics and based on classification codes under the UK Socio-Economic Occupation Classification, SOC 2000) [23].

Sensitivity analysis was carried out and had three main foci: First, cost-utility analysis was conducted from a UK NHS perspective by excluding private health care costs. Second, as there is a lack of consensus with respect to methods for conducting economic evaluation alongside factorial trials, an alternative approach ('within the table') to analysing the factorial trial treatment options was undertaken. The 'within the table' approach assumes that there is an interaction between the treatments, and considers all four arms of the trial as separate interventions [8-9]. With this analysis, the interventions were ordered in terms of increasing QALYseost and compared incrementally. The most cost-effective option was selected based on the principles of dominance (where an intervention is less costly and more effective than the comparator(s)) and extended (weak) dominance (where an intervention is ruled out if the ICER is greater than that of a more effective intervention). Third, cost-utility analysis was conducted using EQ-5D scores generated from the EQ-5D-5L tariff published by Devlin et al. $2018[24]$

\section{Results}

A total of 256 participants were randomised (64 per arm) to one of the four intervention arms. The mean age of participants across all arms was 53.8 years (S.D. 10.2) and 52\% of participants were female. The mean (SD) total Shoulder Pain and Disability Index (SPADI) score at baseline was 61.1 (18.1). Full details of other baseline characteristics can be found elsewhere [25]. 


\section{Resource use and costs}

Complete resource use data were obtained for 194 participants $(76 \%)$. This ranged from $72 \%$ in the ultrasound-guided injection and physiotherapist-led exercise arm to $78 \%$ in the unguided injection and advice and exercise leaflet arm. A full breakdown of patient responses at each follow-up time point can be found elsewhere [25]. Compared to the advice and exercise leaflet group, significantly fewer visits to the GP were made during the 12 month follow-up period in the physiotherapist-led exercise group. The unguided injection group made more visits to a hospital nurse and specialist over the 12 month follow-up period compared to the ultrasoundguided injection group (Supplementary Table 2). With the exception of diclofenac which was significantly lower in the physiotherapist-led exercise arm, there was no significant difference in prescription medication use across trial arms (Supplementary Table 3). Private health care resource use is presented in Supplementary Table 4.

Mean NHS and private costs per participant by trial arm over the 12-month period prior to imputation are presented in Table 1. Health care costs were higher in the physiotherapist-led exercise group compared to the advice and exercise leaflet group and also in the ultrasoundguided injection group compared to the unguided injection group.

\section{Health outcomes}

Health outcomes (EQ-5D and QALYs) using the imputed dataset are presented in table 2. A total of $131(54 \%)$ participants provided complete EQ-5D responses at all time-points. This ranged from $47 \%$ in the unguided injection and physiotherapist-led exercise arm to $61 \%$ in the ultrasound-guided injection and physiotherapist-led exercise arm. However, it should be noted that the majority of those with missing values were missing just one EQ-5D score. There was an improvement in health outcomes over time, with scores obtained at 12-months higher than the baseline scores in all four arms. With respect to QALYs over the 12-month period, higher scores were obtained in the physiotherapist-led exercise and ultrasound-guided injection 
groups when compared to the advice and exercise leaflet and unguided injection groups, respectively. After adjusting for baseline utility, similar results were obtained.

\section{Cost-utility analysis}

The results from the cost-effectiveness analysis using the imputed dataset showed that physiotherapist-led exercise was more expensive (cost difference £155.99) and more effective (QALY difference 0.031) than the advice and exercise leaflet. The resulting ICER was $£ 5,031$ per QALY gained (Table 3) with an 85\% chance of physiotherapist-led exercise being costeffective at a cost-effectiveness threshold of $£ 20,000$ per QALY gained (Figures 1 and 2). Ultrasound-guided injection was also more expensive (cost difference £15.89) and more effective (QALY difference 0.024 ) than unguided injection. The resulting ICER was $£ 662$ per QALY gained (Table 3) with an $83 \%$ chance of ultrasound-guided injection being costeffective at a willingness to pay threshold of $£ 20,000$ per QALY gained (Figures 1 and 2).

\section{Time off work and Productivity Losses}

Participants in the advice and exercise leaflet and unguided injection groups took more time off work than those in the physiotherapist-led exercise and ultrasound-guided injection groups. Productivity losses in terms of lost wages were also higher in the advice and exercise leaflet and unguided injection groups. However, these differences were not statistically significant (Supplementary Table 4 and Table 1).

\section{Sensitivity Analysis}

From a UK NHS perspective, the results obtained were similar to the base case analysis i.e. physiotherapist-led exercise was cost-effective compared to providing an advice and exercise leaflet and ultrasound-guided injection was cost-effective compared to unguided injection (Table 3). Results from the 'within the table' analysis showed that a combination of ultrasoundguided injection and physiotherapist-led exercise was the most cost-effective intervention 
( $£ 6,225$ per QALY gained 10,583 per QALY gained), supporting findings from the base case analysis (Table 4). The results obtained when the EQ-5D-5L tariff [24] was used are similar to those obtained with the mapping algorithm [16] and support the finding that physiotherapistled exercise is cost-effective compared to the advice and exercise leaflet, and ultrasound-guided injection is cost-effective when compared to unguided injection (Supplementary Tables 5 and 6).

\section{Discussion}

\section{Summary of the main findings}

This health economic evaluation showed that for the management of SAPS, physiotherapistled exercise was cost-effective compared to the advice and exercise leaflet and ultrasoundguided injection was cost-effective when compared to unguided injection. Sensitivity analysis using the within-the-table analysis yielded similar results to the at-the-margins approach indicating that the choice of method did not change the findings of the health economic analysis. Similar findings with respect to the choice of method have been obtained in previous studies [8]. A previous economic evaluation showed that a combination of injection and exercise was cost-effective compared to exercise alone [2]. The results from this study add to this evidence by showing that the approach to providing exercise (physiotherapist-led, individualised, progressed and supervised exercise rather than through a standardised advice and exercise leaflet) and injection (guided using ultrasound rather than unguided) is important in terms of increasing their cost-effectiveness.

The results of the trial on which the economic evaluation is based showed that physiotherapistled exercise was more effective than an exercise leaflet whilst ultrasound-guidance conferred no additional benefit over unguided corticosteroid injection [25]. The health economic results are similar in terms of the comparison between physiotherapist-led exercise and the advice and 
exercise leaflet, but different in terms of the comparison between ultrasound-guided and unguided injection. It is however important to note that the primary outcome of the clinical trial was the Shoulder Pain and Disability Index whilst that of the economic evaluation was total QALYs obtained from EQ-5D. Although the difference in QALYs obtained for both comparisons was very small and not statistically significant, the agreed approach in health economics is to conduct a cost-effectiveness analysis with costs and effects estimated jointly [26]. Whilst clinical research focuses on hypothesis testing, economic evaluation is aimed at estimation which may produce contradictory results as was seen in this study with the injection comparison [27]. An important issue which the results of this study raise relates to whether the use of generic quality of life measures such as the EQ-5D are sufficient for assessing the costeffectiveness of interventions in this clinical syndrome. Previous research has shown that generic quality of life measures may be insensitive to changes in some disease areas including musculoskeletal conditions such as osteoarthritis (OA) [28-30].

\section{Strengths and weaknesses of the study}

A strength of the study is the factorial nature of the trial, which enabled us to compare four interventions within the same analysis.

A possible limitation of the economic analysis is the fact that the cost of training clinicians to perform ultrasound-guided injection was not included in the analysis and it could be argued that this may have under-estimated the cost of the ultrasound-guided injection. The cost and time required to train clinical staff to perform ultrasound-guided injections is a potential barrier to services. However, it should be noted that there are a number of difficulties associated with the estimation of a per patient training cost within economic evaluation studies and also the training received by clinicians would be used for a large number of patients over a number of years which would result in a low mean cost per patient. A further limitation was that only $76 \%$ 
of participants had complete data at all time-points, however, the missing data were accounted for using multiple imputations.

\section{Meaning of the results}

This health economic analysis has shown that physiotherapist-led exercise and ultrasoundguided injection are more cost-effective treatment options for SAPS than an advice and exercise leaflet or unguided shoulder injection respectively. However, this conclusion should be interpreted in the light of the clinical results which showed that physiotherapist-led exercise conferred superior benefits for patients compared to the advice and leaflet yet ultrasound guided injection conferred no additional benefit over unguided injection [25].

Acknowledgements: This project was undertaken with the support of Keele Clinical Trials Unit, Keele University, UK. The authors would like to thank Kris Clarkson, Treena Larkin, and Sue Weir. We would also like to thank the clinicians, managerial and administrative staff of the Midlands Partnership NHS Foundation Trust, and members of the trial steering committee and data monitoring committee.

Funding: This paper presents independent research funded by the National Institute for Health Research (NIHR) under its Research for Patient Benefit programme (Grant Reference Number PB-PG-1207-15064) and the Arthritis Research UK Primary Care Centre grant (Grant Number 18139). NEF and EMH are both NIHR Senior Investigators. NEF was supported through an NIHR Research Professorship (NIHR-RP- 011-015). The views expressed are those of the authors and not necessarily those of the NHS, the NIHR or the Department of Health and Social Care. 
Conflicts of interest: None to declare

\section{References}

1. Urwin M, Symmons D, Allison T et al. Estimating the burden of musculoskeletal disorders in the community: the comparative prevalence of symptoms at different anatomical sites, and the relation to social deprivation. Ann Rheum Dis 1998;57:649-55.

2. Jowett S, Crawshaw DP, Helliwell PS, Hensor EM, Hay EM, Conaghan PG. Costeffectiveness of exercise therapy after corticosteroid injection for moderate to severe shoulder pain due to subacromial impingement syndrome: a trial-based analysis. Rheumatology. 2013;52:1485-91.

3. Luime JJ, Koes BW, Hendriksen IJ, Burdorf A, Verhagen AP, Miedema HS, Verhaar JA. Prevalence and incidence of shoulder pain in the general population; a systematic review. Scand J Rheumatol. 2004;33:73-81.

4. Van der Windt DA, Koes BW, de Jong BA, Bouter LM. Shoulder disorders in general practice: incidence, patient characteristics, and management. Annals of the rheumatic diseases. Ann Rheum Dis 1995;54:959-64.

5. Hanchard N, Cummins J, Jeffries J. Evidence-Based Clinical Guidelines for the Diagnosis, Assessment and Physiotherapy Management of Shoulder Impingement Syndrome. London: Chartered Society of Physiotherapy, 2004.

6. Kulkarni R, Gibson J, Brownson P, Thomas M, Rangan A, Carr AJ, et al. Subacromial shoulder pain. Shoulder \& Elbow. 2015;7:135-43.

7. Roddy E, Zwierska I, Hay EM, Jowett S, Lewis M, Stevenson K, et al. Subacromial impingement syndrome and pain: protocol for a randomised controlled trial of exercise and corticosteroid injection (the SUPPORT trial). BMC Musculoskelet Disord. 2014;15:81.

8. Dakin H, Gray A 2017 Economic evaluation of factorial randomised controlled trials: challenges, methods and recommendations Stat Med 2017;36:2814-2830

9. Oppong R, Jowett S, Nicholls E, Whitehurst DG, Hill S, Hammond A, et al. Joint protection and hand exercises for hand osteoarthritis: an economic evaluation comparing methods for the analysis of factorial trials. Rheumatology. 2014;54:876-83.

10. Curtis L. Unit Costs of Health and Social Care 2012 PSSRU. University of Kent: Canterbury. 2012.

11. BMJ Group. British National Formulary (BNF) 66. London: BMJ Group and RPS Publishing . 2012.

12. NHS reference cost 2012/2013 https://www.gov.uk/government/publications/nhsreference-costs-2012-to-2013 (accessed 16/07/2018) 
13. Stevenson, K., Jackson, S., Shufflebotham, J., Roddy, E. and Foster, N.E., Development and delivery of a physiotherapist-led exercise intervention in a randomised controlled trial for subacromial impingement syndrome (the SUPPORT trial). Physiotherapy, 2017;103:379-386.

14. Herdman M, Gudex C, Lloyd A, Janssen MF, Kind P, Parkin D, et al. Development and preliminary testing of the new five-level version of EQ-5D (EQ-5D-5L). Qual Life Res. 2011;20:1727-36.

15. NICE: Position statement on use of the EQ-5D-5L valuation set 2017 https://www.nice.org.uk/about/what-we-do/our-programmes/nice-guidance/technologyappraisal-guidance/eq-5d-51 (accessed 20/02/2019)

16. Van Hout B, Janssen MF, Feng YS, Kohlmann T, Busschbach J, Golicki D, et al. Interim scoring for the EQ-5D-5L: mapping the EQ-5D-5L to EQ-5D-3L value sets. Value in health. 2012 Jul 1;15(5):708-15.

17. Rubin DB. Multiple imputation for nonresponse in surveys. New York, USA: John Willey \& Sons 1987.

18. White IR, Royston P, Wood AM. Multiple imputation using chained equations: Issues and guidance for practice Stat Med 2011; 30: 337-99.

19. Matthews J, Altman DG, Campbell MJ, Royston P. Analysis of serial measurements in medical research. BMJ 1990;300:230-5.

20. Manca A, Hawkins N, Sculpher MJ. Estimating mean QALYs in trial based cost effectiveness analysis: the importance of controlling for baseline utility. Health Econ. 2005;14:487-496.

21. Van Hout BA, Al MJ, Gordon GS, Rutten FF. Costs, effects and C/E-ratios alongside a clinical trial. Health Econ 1994;3:309-19.

22. STATA 2012 StataCorp. LP. Stata/IC 12.1 for Windows. 2012

23. Standard Occupational Classification (SOC) 2000 http://www.ons.gov.uk/ons/guidemethod/classifications/archived-standard-classifications/standard-occupational-classification2000/about-soc-2000/index.html

24. Devlin NJ, Shah KK, Feng Y, Mulhern B, van Hout B. Valuing health-related quality of life: An EQ-5 D-5 L value set for E ngland. Health economics. 2018 Jan;27(1):7-22.

25. Roddy E, Ogollah R, Oppong R, Zwierska I, Datta P, Hall A et al. Optimising the outcome of exercise and corticosteroid injection in patients with subacromial impingement syndrome: a factorial randomised trial Br J Sports Med http://dx.doi.org/10.1136/bjsports2019-101268

26. Dakin H, Wordsworth S. Cost-Minimisation Analysis Versus Cost-Effectiveness Analysis, Revisited. Health Econ 2013;22:22-34 
27. Whitehurst DGT, Bryan S Trial-based and economic analyses: the unhelpful quest for conformity. Trials 2013;14:421

28. National Institute for Health and Care Excellence. Guide to the Methods of Technology Appraisal. London, UK: National Institute for Health and Care Excellence, 2013.

29. Brazier JE, Harper R, Munro J, Walters SJ, Snaith ML. Generic and condition-specific outcome measures for people with osteoarthritis of the knee. Rheumatology 1999;38:870-7.

30. Oppong R, Jowett S, Nicholls E, Whitehurst DG, Hill S, Hammond A, et al. Joint protection and hand exercises for hand osteoarthritis: an economic evaluation comparing methods for the analysis of factorial trials. Rheumatology. 2014;54:876-83. 
Table 1: Mean health care cost (SD) per patient over 12 months (complete cases) ( $£, 2019$ prices)

\begin{tabular}{|c|c|c|c|c|c|c|}
\hline & \multicolumn{3}{|c|}{ Injection intervention group } & \multicolumn{3}{|c|}{ Exercise intervention group } \\
\hline & $\begin{array}{l}\text { US-guided } \\
\quad(n=95)\end{array}$ & $\begin{array}{c}\text { Unguided } \\
(\mathrm{n}=99)\end{array}$ & $\begin{array}{c}\text { Mean difference } \\
(95 \% \mathrm{CI})\end{array}$ & $\begin{array}{c}\begin{array}{c}\text { Physiotherapist- } \\
\text { led } \\
(\mathrm{n}=95)\end{array} \\
\end{array}$ & $\begin{array}{c}\begin{array}{c}\text { Advice and Exercise } \\
\text { Leaflet } \\
(\mathbf{n}=99)\end{array} \\
\end{array}$ & $\begin{array}{c}\text { Mean difference } \\
(95 \% \text { CI })\end{array}$ \\
\hline Intervention $\operatorname{cost} \mathrm{t}^{\mathrm{a}}$ & $\begin{array}{l}186.01 \\
(126.50)\end{array}$ & $\begin{array}{l}107.12 \\
(127.33)\end{array}$ & $\begin{array}{c}78.89 \\
(41.98,113.59)\end{array}$ & $258.92(96.73)$ & $37.15(36.58)$ & $\begin{array}{c}221.77 \\
(196.45,241.00)\end{array}$ \\
\hline $\begin{array}{l}\text { PRIMARY CARE } \\
\text { CONTACTS }\end{array}$ & & & & & & \\
\hline General Practitioner & $39.16(75.27)$ & $43.30(97.90)$ & $\begin{array}{c}-4.14 \\
(-28.39,20.59)\end{array}$ & $28.01(70.66)$ & $53.99(99.52)$ & $\begin{array}{c}-25.98 \\
(-49.65,-3.08)\end{array}$ \\
\hline Practice Nurse & $1.28(11.11)$ & $0.58(4.16)$ & $\begin{array}{c}0.70 \\
(-0.84,3.99)\end{array}$ & $1.21(9.73)$ & $0.92(6.73)$ & $\begin{array}{c}0.29 \\
(-1.69,3.46)\end{array}$ \\
\hline $\begin{array}{l}\text { Other professionals in } \\
\text { GP practice } \\
\text { SECONDARY CARE } \\
\text { CONTACTS }\end{array}$ & $14.48(40.98)$ & $22.58(59.73)$ & $\begin{array}{c}-8.10 \\
(-22.99,4.19)\end{array}$ & $24.52(58.25)$ & $12.94(43.47)$ & $\begin{array}{c}11.58 \\
(-2.64,26.78)\end{array}$ \\
\hline Physiotherapist ${ }^{b}$ & $37.27(86.59)$ & $32.69(86.02)$ & $\begin{array}{c}4.58 \\
(-20.45,27.03)\end{array}$ & $53.31(102.03)$ & $17.30(63.03)$ & $\begin{array}{c}36.01 \\
(14.52,62.41)\end{array}$ \\
\hline NHS consultants & $26.97(60.56)$ & $49.08(93.36)$ & $\begin{array}{c}-22.11 \\
(-43.75,-1.21)\end{array}$ & $38.15(74.24)$ & $38.34(76.12)$ & $\begin{array}{c}-0.19 \\
(-23.44,21.53)\end{array}$ \\
\hline $\begin{array}{l}\text { Private consultants and } \\
\text { Physiotherapists }\end{array}$ & $18.07(83.84)$ & $8.98(35.80)$ & $\begin{array}{c}9.09 \\
(-5.96,30.28)\end{array}$ & $6.17(27.97)$ & $20.40(84.94)$ & $\begin{array}{c}-14.23 \\
(-38.09,-0.79)\end{array}$ \\
\hline $\begin{array}{l}\text { Other professional in } \\
\text { NHS hospitals } \\
\text { REPEAT INJECTIONS }\end{array}$ & $1.58(11.87)$ & $2.07(11.14)$ & $\begin{array}{c}-0.49 \\
(-3.61,2.93)\end{array}$ & $1.46(11.81)$ & $2.20(11.19)$ & $\begin{array}{c}-0.74 \\
(-3.82,2.81)\end{array}$ \\
\hline Repeat Injections in NHS & $35.96(98.07)$ & $51.07(118.07)$ & $\begin{array}{c}-15.11 \\
(-45.35,17.64)\end{array}$ & $33.08(104.93)$ & $53.83(111.82)$ & $\begin{array}{c}-20.75 \\
(-50.81,8.58)\end{array}$ \\
\hline $\begin{array}{l}\text { Repeat Injections in } \\
\text { private practice } \\
\text { DIAGNOSTIC TESTS }\end{array}$ & $1.43(14.02)$ & - & $\begin{array}{c}1.43 \\
(0,5.88)\end{array}$ & - & $1.38(13.73)$ & $\begin{array}{c}-1.38 \\
(-5.11,0)\end{array}$ \\
\hline Diagnostic tests in NHS & $36.27(105.24)$ & $72.97(144.77)$ & $\begin{array}{c}-36.70 \\
(-70.06,-5.69)\end{array}$ & $45.44(114.17)$ & $64.18(139.89)$ & $\begin{array}{c}-18.74 \\
(-53.69,17.34)\end{array}$ \\
\hline $\begin{array}{l}\text { Diagnostic tests in private } \\
\text { practice }\end{array}$ & - & $1.76(12.98)$ & $\begin{array}{c}-1.76 \\
(-5.38,0)\end{array}$ & - & $1.76(12.98)$ & $\begin{array}{c}-1.76 \\
(-4.96,0)\end{array}$ \\
\hline
\end{tabular}




\section{MEDICATION}

Prescribed medication

$\begin{array}{lcc}5.70(16.62) & 7.80(16.96) & -2.10 \\ & & (-6.74,2.51) \\ 10.15(20.01) & 18.86(57.93) & -8.71\end{array}$

$3.71(7.19)$

$9.71(22.08)$

$-6.00$

Over the counter medication

$10.15(20.01) \quad 18.86(57.93)$

$(-22.80,1.11)$

$13.85(45.62)$

$15.31(42.19)$

$(-10.86,-1.78)$

$-1.46$

\section{WORK RELATED \\ OUTCOMES}

Productivity costs

388.06

823.56

$-435.50$

$562.54(2713.56) \quad 656.13(3317.80)$

$(-12.94,11.17)$

\section{TOTAL COSTS}

(1638.82)

(3924.91)
$(-1472.23,239.61)$

$4.70,654.54)$

\begin{tabular}{lllc} 
Total health care $\operatorname{costs}^{\mathrm{c}}(\mathrm{n}=256)$ & 428.01 & 412.12 & 15.89 \\
& $(322.83)$ & $(366.09)$ & $(-59.36,109.86)$ \\
\hline
\end{tabular}

${ }^{a}$ This cost takes into account the fact that participants had more the intervention have been excluded ${ }^{\mathrm{c}}$ Costs have been imputed 
Table 2: Mean health outcomes (SD) over 12 months (imputed data)

\begin{tabular}{|c|c|c|c|c|c|c|}
\hline & \multicolumn{3}{|c|}{ Injection intervention group } & \multicolumn{3}{|c|}{ Exercise intervention group } \\
\hline & $\begin{array}{l}\text { US-guided } \\
(n=128)\end{array}$ & $\begin{array}{l}\text { Unguided } \\
(n=128)\end{array}$ & $\begin{array}{c}\text { Mean difference } \\
(95 \% \mathrm{CI})\end{array}$ & $\begin{array}{l}\text { Physiotherapist-led } \\
(n=128)\end{array}$ & $\begin{array}{c}\text { Advice \& exercise leaflet } \\
(\mathrm{n}=128)\end{array}$ & $\begin{array}{l}\text { Mean difference } \\
(95 \% \mathrm{CI})\end{array}$ \\
\hline \multicolumn{7}{|l|}{ EQ-5D scores } \\
\hline Baseline & $0.494(0.255)$ & $0.501(0.235)$ & $-0.007(-0.065,0.052)$ & $0.504(0.243)$ & $0.492(0.247)$ & $0.012(-0.042,0.078)$ \\
\hline 6 weeks & $0.599(0.240)$ & $0.574(0.236)$ & $0.025(0.031,0.080)$ & $0.601(0.242)$ & $0.572(0.234)$ & $0.029(-0.025,0.087)$ \\
\hline 6 months & $0.577(0.245)$ & $0.549(0.261)$ & $0.028(-0.031,0.088)$ & $0.584(0.246)$ & $0.541(0.259)$ & $0.043(-0.018,0.103)$ \\
\hline 12 months & $0.599(0.248)$ & $0.594(0.271)$ & $0.005(-0.058,0.066)$ & $0.606(0.268)$ & $0.588(0.252)$ & $0.018(-0.052,0.081)$ \\
\hline Total QALYs & $0.584(0.225)$ & $0.564(0.230)$ & $0.020(-0.035,0.072)$ & $0.594(0.227)$ & $0.555(0.226)$ & $0.039(-0.013,0.097)$ \\
\hline $\begin{array}{l}\text { Total adjusted } \\
\text { QALYs a }\end{array}$ & 0.586 & 0.562 & $0.024(-0.018,0.066)$ & 0.589 & 0.558 & $0.031(-0.010,0.073)$ \\
\hline
\end{tabular}

${ }^{a}$ QALYs adjusted for baseline utility 
Table 3: Cost-utility analysis (imputed data)

\begin{tabular}{|c|c|c|c|c|c|c|}
\hline & \multicolumn{2}{|c|}{ Injection intervention group } & & \multicolumn{3}{|c|}{ Exercise intervention group } \\
\hline & $\begin{array}{l}\text { US-guided } \\
(\mathrm{n}=128)\end{array}$ & $\begin{array}{l}\text { Unguided } \\
(n=128)\end{array}$ & & $\begin{array}{l}\text { Physiotherapist-led } \\
(\mathrm{n}=128)\end{array}$ & $\begin{array}{c}\begin{array}{c}\text { Advice \& Exercise } \\
\text { Leaflet } \\
(\mathbf{n}=\mathbf{1 2 8})\end{array} \\
\end{array}$ & \\
\hline & Mean & Mean & $\begin{array}{l}\text { Mean difference } \\
\text { (Confidence } \\
\text { Interval) }\end{array}$ & Mean & Mean & $\begin{array}{l}\text { Mean difference } \\
\text { (Confidence } \\
\text { Interval) }\end{array}$ \\
\hline \multicolumn{7}{|c|}{ HEALTHCARE PERSPECTIVE } \\
\hline Cost & $£ 428.01$ & $£ 412.12$ & $\begin{array}{c}£ 15.89 \\
(-59.36,109.86)\end{array}$ & $£ 498.06$ & $£ 342.07$ & $\begin{array}{c}£ 155.99 \\
(69.02,241.93)\end{array}$ \\
\hline QALYs $^{\text {a }}$ & 0.586 & 0.562 & $\begin{array}{c}0.024 \\
(-0.018,0.066)\end{array}$ & 0.589 & 0.558 & $\begin{array}{c}0.031 \\
(-0.010,0.073)\end{array}$ \\
\hline ICER & $£ 662$ per QALY gained & & & $£ 5,031$ per QALY gained & & \\
\hline \multicolumn{7}{|c|}{ NHS PERSPECTIVE } \\
\hline Cost & $£ 398.25$ & $£ 382.04$ & $\begin{array}{c}£ 15.85 \\
(-64.32,90.22)\end{array}$ & $£ 476.02$ & $£ 304.27$ & $\begin{array}{c}£ 171.75 \\
(98.44,251.88)\end{array}$ \\
\hline QALYs ${ }^{a}$ & 0.586 & 0.562 & $\begin{array}{c}0.024 \\
(-0.018,0.066)\end{array}$ & 0.589 & 0.558 & $\begin{array}{c}0.031 \\
(-0.010,0.073)\end{array}$ \\
\hline ICER & $£ 660$ per QALY gained & & & $£ 5,540$ per QALY gained & & \\
\hline
\end{tabular}


Table 4: Cost-utility analysis "within the table" analysis

\begin{tabular}{|c|c|c|c|c|}
\hline & Cost (£)* & QALY & QALY*a & $\underline{\text { ICER }}$ \\
\hline \multicolumn{5}{|c|}{ Health care perspective } \\
\hline $\begin{array}{l}\text { Unguided injection and advice and } \\
\text { exercise leaflet }(n=64)\end{array}$ & $\underline{385.31(397.70)}$ & $\underline{0.545(0.236)}$ & $\underline{0.542}$ & $\begin{array}{l}\text { Dominated by Ultrasound-guided injection and } \\
\text { advice and exercise leaflet }{ }^{\text {b }}\end{array}$ \\
\hline $\begin{array}{l}\text { Ultrasound-guided injection and advice } \\
\text { and exercise leaflet }(\mathrm{n}=64)\end{array}$ & $298.82(281.99)$ & $\underline{0.564(0.218)}$ & $\underline{0.575}$ & $\begin{array}{l}\text { Dominates Unguided injection and advice and } \\
\underline{\text { exercise leaflet }}{ }^{\mathrm{b}}\end{array}$ \\
\hline $\begin{array}{l}\text { Unguided injection and } \\
\text { Physiotherapist-led exercise }(n=64)\end{array}$ & $\underline{438.92(332.49)}$ & $\underline{0.583(0.224)}$ & $\underline{0.582}$ & $\underline{£ 20,014 \text { per QALY gained }}{ }^{c}$ \\
\hline Ultrasound-guided injection and & $\underline{557.20(310.90)}$ & $\underline{0.604(0.232)}$ & $\underline{0.601}$ & $£ 6,225.26$ per QALY gained ${ }^{\mathrm{d}}$ \\
\hline
\end{tabular}

NHS perspective

\begin{tabular}{|c|c|c|c|c|}
\hline & $\underline{\text { Cost* }}$ & QALY & $\underline{\text { QALY*a }}$ & $\underline{\text { ICER }}$ \\
\hline Unguided injection and advice and & $\underline{347.87(385.17)}$ & $\underline{0.545(0.236)}$ & $\underline{0.542}$ & Dominated by Ultrasound-guided injection and \\
\hline exercise leaflet $(n=64)$ & & & & $\underline{\text { advice } \text { and exercise leaflet }}{ }^{\mathrm{b}}$ \\
\hline Ultrasound-guided injection and advice & $\underline{260.66(220.67)}$ & $\underline{0.564(0.218)}$ & $\underline{0.575}$ & Dominates Unguided injection and advice and \\
\hline and exercise leaflet $(n=64)$ & & & & $\underline{\text { exercise leaflet }}$ b \\
\hline Unguided injection and & $\underline{416.21(324.81)}$ & $\underline{0.583(0.224)}$ & $\underline{0.582}$ & $£ 22,221.43$ per QALY gained ${ }^{c}$ \\
\hline
\end{tabular}

$\underline{\text { Physiotherapist-led exercise }(\mathrm{n}=64)}$ 


\section{Ultrasound-guided injection and}

$\underline{535.83(305.61)}$

$\underline{0.604(0.232)}$

$\underline{0.601}$

$\underline{£ 6,295 \text { per QALY gained }}{ }^{\mathrm{d}}$

\section{Physiotherapist-led exercise $(\mathrm{n}=64)$}

*Cost and QALYs imputed a Adjusted for baseline utility ${ }^{\mathrm{b}}$ Ultrasound-guided injection and advice and exercise leaflet versus Unguided injection and advice and exercise leaflet ${ }^{\mathrm{c}}$ Unguided injection and Physiotherapist-led exercise versus Ultrasound-guided injection and advice and exercise leaflet ${ }^{\mathrm{d}}$ Ultrasound-guided injection and

Physiotherapist-led exercise versus Unguided injection and Physiotherapist-led exercise 
Figure 1: Cost-effectiveness plane

\section{Cost-effectiveness plane}
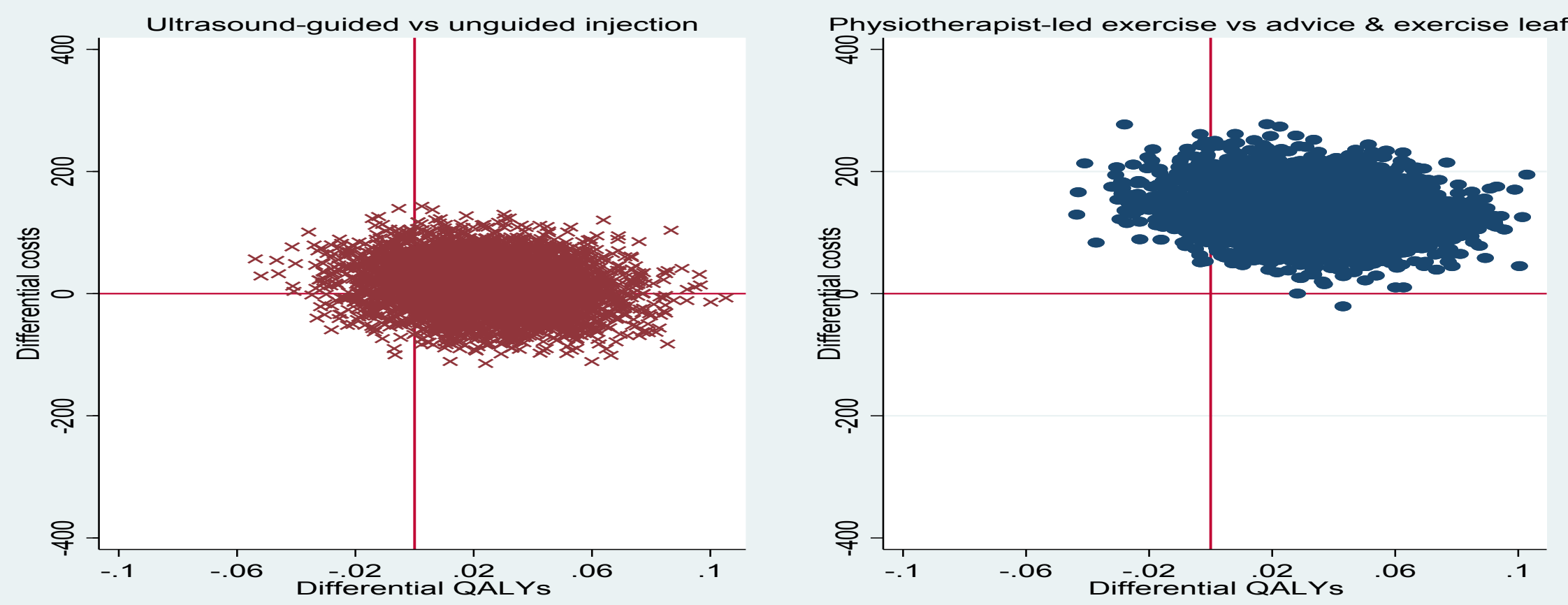
Figure 2: Cost-effectiveness acceptability curve

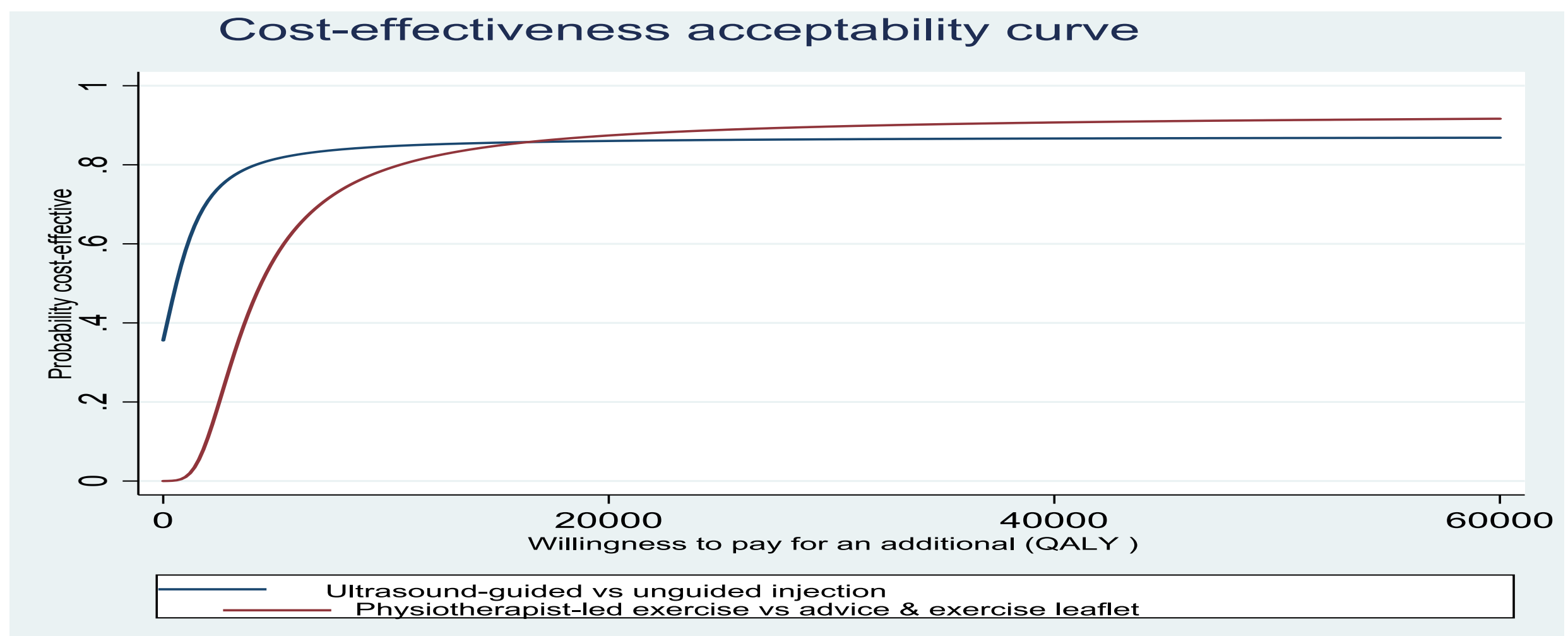




\title{
The cost-effectiveness of different approaches to exercise and corticosteroid injection for Subacromial pain (impingement) syndrome
}

\begin{abstract}
Authors:
Raymond Oppong ${ }^{1,4}$, Sue Jowett ${ }^{1,4}$, Martyn Lewis ${ }^{1,3}$, Edward Roddy ${ }^{1,2}$, Reuben O Ogollah ${ }^{1,3}$, Irena Zwierska $^{1,3}$, Praveen Datta ${ }^{5}$, Alison Hall ${ }^{1}$, Elaine Hay ${ }^{1,2}$, Julie Shufflebotham ${ }^{1,6}$, Kay Stevenson ${ }^{1,2}$, Danielle A van der Windt ${ }^{1}$, Julie Young ${ }^{1,3}$, Nadine E Foster ${ }^{1,3}$.
\end{abstract}

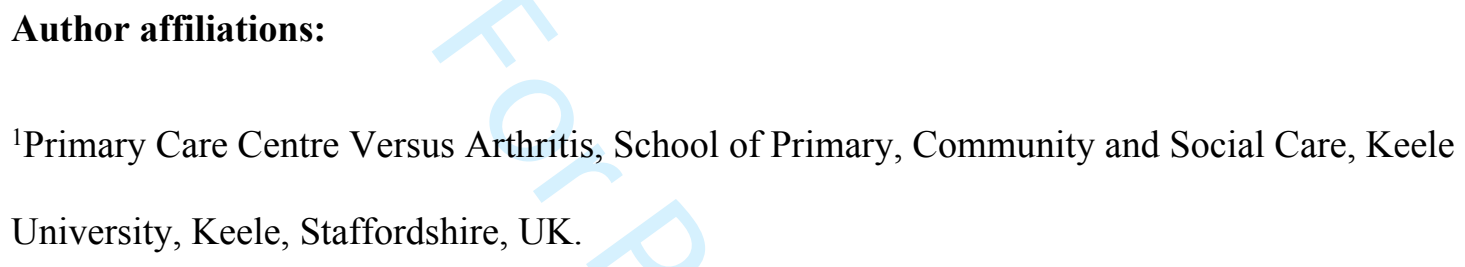

${ }^{6}$ Midlands Partnership NHS Foundation Trust, Newcastle-under-Lyme, Staffordshire, UK

\section{Corresponding author:}

Dr Sue Jowett, Health Economics Unit, Institute of Applied Health Research, University of Birmingham, B15 2TT

e-mail: s.jowett@bham.ac.uk

ORCID iD: 0000-0001-8936-3745 


\begin{abstract}
:
Objectives: To determine whether physiotherapist-led exercise intervention and ultrasoundguided subacromial corticosteroid injection is cost-effective when compared to standard advice and exercise leaflet and unguided injection in patients with subacromial pain (impingement) syndrome.
\end{abstract}

Methods: An incremental cost-utility analysis using patient responses to the five-level EuroQoL-5D (EQ-5D-5L) questionnaire was undertaken from a health care perspective alongside a 2x2 factorial randomised trial with 256 participants over a 12-month follow-up period. Uncertainty was explored through the use of cost-effectiveness acceptability curves.

Results: The cost-utility analysis indicated that physiotherapist-led exercise was associated with an incremental cost of $£ 155.99$ (95\% CI: 69.02 to 241.93 ) and 0.031 (95\% CI: -0.01 to 0.07) additional quality-adjusted life-years (QALYs), an incremental cost-effectiveness ratio (ICER) of $£ 5,031$ per QALY gained and an $85 \%$ chance of being cost-effective at a threshold of $£ 20,000$ per QALY gained compared to the advice and exercise leaflet. Ultrasound-guided injection was associated with an incremental cost of $£ 15.89$ (95\% CI: -59.36 to 109.86$)$ and 0.024 (95\% CI: -0.02 to 0.07$)$ additional QALYs, an ICER of $£ 662$ per QALY gained and a $83 \%$ chance of being cost-effective at a threshold of $£ 20,000$ per QALY gained compared to unguided injection.

Conclusion: Physiotherapist-led exercise was cost-effective compared to the advice and exercise leaflet, and ultrasound-guided injection was cost-effective when compared to unguided injection.

Clinical trial registration number: ISRCTN (42399123)

Keywords: Cost-effectiveness, Subacromial pain (impingement) syndrome , Exercise, Corticosteroid injection, EQ-5D 
Rheumatology key messages:

- Guidelines recommend non-surgical options for the management of Subacromial pain (impingement) syndrome.

- Physiotherapist-led exercise and ultrasound-guided corticosteroid injection appear to increase costs but lead to improved quality of life compared to unguided injection and advice and exercise leaflet.

- Physiotherapist-led exercise and ultrasound-guided injection are likely to be costeffective. 


\section{Introduction}

Subacromial pain (impingement) syndrome (SAPS) is the most common cause of shoulder pain. The prevalence of SAPS increases with age and places a substantial burden on health systems, with estimates from GP consultations suggesting that approximately $16 \%$ of adults reporting musculoskeletal pain had shoulder pain [1-2]. Shoulder pain poses a considerable burden to society, accounting for a substantial number of days off work and associated productivity costs [3-4]. Treatment guidelines recommend initial non-surgical management including education/self-management advice, exercise, corticosteroid injection, and analgesia $[5-6]$.

In order to explore how to optimise outcomes from exercise and subacromial corticosteroid injection, a randomised controlled trial was conducted to assess whether better improvements in pain and function were obtained with (1) a physiotherapist-led, individualised, supervised and progressed exercise programme versus a standardised advice and exercise leaflet, and (2) ultrasound-guided subacromial corticosteroid injection versus unguided injection. The clinical results of the trial showed that physiotherapist-led exercise was more effective than an exercise leaflet whilst ultrasound-guidance conferred no additional benefit over unguided corticosteroid injection [7]. In addition to establishing the effectiveness of interventions for the management of shoulder pain, it is also important to determine whether these interventions offer value for money. Previous studies assessing the cost-effectiveness of interventions for the treatment of SAPS have focused on a comparison of corticosteroid injection and exercise [2] and have not compared different methods of guiding corticosteroid injection, nor the manner in which the exercise intervention was delivered.

The primary aim of this economic analysis was to determine whether a physiotherapist-led exercise intervention is cost-effective compared to a standard advice and exercise leaflet, and 
whether ultrasound-guided subacromial corticosteroid injection is cost-effective when compared to unguided injection.

\section{Methods}

A within-trial economic evaluation was conducted alongside a $2 \times 2$ factorial randomised trial in patients with SAPS [7]. Using block randomisation, participants who met the inclusion criteria were randomly assigned on a 1:1:1:1 basis to one of four interventions:

1. Ultrasound-guided subacromial corticosteroid injection and physiotherapist-led individualised, supervised and progressed exercise.

2. Ultrasound-guided subacromial corticosteroid injection and an advice and exercise leaflet.

3. Unguided subacromial corticosteroid injection and physiotherapist-led individualised, supervised and progressed exercise

4. Unguided subacromial corticosteroid injection and an advice and exercise leaflet.

The main outcome for the economic analysis was the quality-adjusted life-year (QALY), over a 12-month follow-up period. The base case analysis was carried out from a health care perspective including health care costs incurred by the NHS and patients. To be consistent with the clinical study, the 'at the margins' approach which assumes that interventions are independent was adopted for the base case scenario [8-9]. The trial, including this economic analysis, was approved by The Black Country Research Ethics Committee (10/H1202/72). Trial registration number (ISRCTN42399123). 


\section{Data collection}

\section{Resource use and costs}

Data on health care resource use were obtained from participant responses to self-report questionnaires administered at 6 weeks, 6 months and 12 months. Resource use data concentrated on visits to health care professionals in primary and secondary care, medical investigations/interventions, and both prescribed and over the counter medications. Data obtained from questionnaire responses were aggregated to generate overall resource use over the 12-month follow-up period. The questionnaires also requested information on time off work over 12 months which was used to estimate productivity losses.

Unit cost data were obtained from the following sources: British National Formulary, NHS reference costs and the PSSRU publication on unit cost of health and social care [10-12] (Supplementary Table 1). 2012/2013 costs were inflated to 2019 prices using the consumer price index.

\section{Trial intervention cost}

Information on the intervention costs was obtained from trial data and all resources required to deliver the interventions were determined.

Physiotherapist-led exercise versus advice and leaflet: For the physiotherapist visits, the unit cost associated with a contact with the physiotherapist was multiplied by the number of physiotherapy contacts. Information on the number of physiotherapist contacts was obtained from case report forms that physiotherapists completed. For the advice and exercise leaflet, only the cost associated with the leaflet was included since all participants had the index consultation where the injection was performed on all patients. Full details of the intervention can be found elsewhere [13]. 
Ultrasound versus unguided injection: It was assumed that since all patients had a similar injection, the only difference between the ultrasound and unguided injection was the cost of the ultrasound. The cost of the ultrasound machine was estimated to be $£ 35,000$, and in order to work out a cost per patient, it was assumed that the life expectancy of the machine is 6 years and that the machine is used about 1872 times a year (this value was obtained through consultation with experts). Applying the annuity factor at an interest rate of 3\%, the cost of the ultrasound machine per patient was $£ 3.45$.

\section{Health Outcomes}

Using participant responses to the 5-level version of the EQ-5D-5L questionnaire [14], quality of life was measured at baseline, 6 weeks, 6 months and 12 months. EQ-5D-5L was mapped back to the EQ-5D 3L valuation set as currently recommended by NICE [15] and used to generate the EQ-5D index scores which were then used to derive QALYs. The mapping algorithm developed by Van Hout et al. 2012 was used for this purpose [16].

\section{Statistical Analysis}

The economic analysis was carried out on an intention to treat basis, with the aim to estimate the difference in costs and QALYs between the physiotherapist-led exercise intervention and the advice and exercise leaflet and between the ultrasound-guided and unguided subacromial corticosteroid injections. Over the 12-month period, missing EQ-5D scores and costs at one or more of the time points were accounted for using multiple imputation methodology to impute 25 separate datasets [17]. The number of datasets were chosen in order to adequately account for the level of missing data in the study. The approach adopted in this study used chained equations with predictive mean matching in order to ensure that the missing values are aligned to the nearest best-fitting values in the dataset [18]. This approach is particularly useful for cost and EQ-5D data which have skewed distributions, and ensures that imputed values do not go 
out of the plausible range. The coefficients were then pooled across the multiply imputed datasets using the Rubin's rule to obtain single estimates of the corresponding population parameters [17].

The imputation model was fitted with gender, age, smoking status at baseline, baseline SPADI score and health status at baseline as predictors. Using the area under the curve approach [19], QALYs over the 12-month period were estimated for each participant. Total health care costs over the 12-month period were calculated by multiplying the resource items by the respective unit cost and summing over all resource use items. Differences in mean costs and QALYs between trial arms were estimated and QALYs were adjusted to control for imbalances in baseline utility between the interventions [20].

Incremental cost effectiveness ratios (ICERs) were estimated by dividing the difference in mean cost between two interventions (physiotherapist-led exercise versus advice and exercise leaflet and ultrasound-guided versus unguided injection) by the difference in mean QALYs. Non-parametric bootstrapping was used to illustrate and quantify uncertainty. To determine the probability of an intervention being deemed cost-effective compared to an alternative intervention (physiotherapist-led exercise versus advice and exercise leaflet and ultrasoundguided versus unguided injection), cost-effectiveness acceptability curves (CEACs) were constructed [21]. This shows the probability that an intervention is cost-effective, relative to the chosen comparator, across a range of values that represent a decision makers' willingness to pay for an additional QALY. All the analyses were carried out in STATA version 12 [22].

Costs for productivity losses were computed through the human capital approach using data collected on time off work and employment. Productivity losses were obtained by the product of lost productivity time and the mean hourly wage of patients classified from data on wages 
corresponding to self-reported baseline occupation (derived from the Annual Surveys of Health Evaluation by the Office of National Statistics and based on classification codes under the UK Socio-Economic Occupation Classification, SOC 2000) [23].

Sensitivity analysis was carried out and had three main foci: First, cost-utility analysis was conducted from a UK NHS perspective by excluding private health care costs. Second, as there is a lack of consensus with respect to methods for conducting economic evaluation alongside factorial trials, an alternative approach ('within the table') to analysing the factorial trial treatment options was undertaken. The 'within the table' approach assumes that there is an interaction between the treatments, and considers all four arms of the trial as separate interventions [8-9]. With this analysis, the interventions were ordered in terms of increasing QALYs and compared incrementally. The most cost-effective option was selected based on the principles of dominance (where an intervention is less costly and more effective than the comparator(s)) and extended (weak) dominance (where an intervention is ruled out if the ICER is greater than that of a more effective intervention). Third, cost-utility analysis was conducted using EQ-5D scores generated from the EQ-5D-5L tariff published by Devlin et al. 2018 [24]

\section{Results}

A total of 256 participants were randomised (64 per arm) to one of the four intervention arms. The mean age of participants across all arms was 53.8 years (S.D. 10.2) and 52\% of participants were female. The mean (SD) total Shoulder Pain and Disability Index (SPADI) score at baseline was 61.1 (18.1). Full details of other baseline characteristics can be found elsewhere [25]. 


\section{Resource use and costs}

Complete resource use data were obtained for 194 participants $(76 \%)$. This ranged from $72 \%$ in the ultrasound-guided injection and physiotherapist-led exercise arm to $78 \%$ in the unguided injection and advice and exercise leaflet arm. A full breakdown of patient responses at each follow-up time point can be found elsewhere [25]. Compared to the advice and exercise leaflet group, significantly fewer visits to the GP were made during the 12 month follow-up period in the physiotherapist-led exercise group. The unguided injection group made more visits to a hospital nurse and specialist over the 12 month follow-up period compared to the ultrasoundguided injection group (Supplementary Table 2). With the exception of diclofenac which was significantly lower in the physiotherapist-led exercise arm, there was no significant difference in prescription medication use across trial arms (Supplementary Table 3). Private health care resource use is presented in Supplementary Table 4.

Mean NHS and private costs per participant by trial arm over the 12-month period prior to imputation are presented in Table 1. Health care costs were higher in the physiotherapist-led exercise group compared to the advice and exercise leaflet group and also in the ultrasoundguided injection group compared to the unguided injection group.

\section{Health outcomes}

Health outcomes (EQ-5D and QALYs) using the imputed dataset are presented in table 2. A total of $131(54 \%)$ participants provided complete EQ-5D responses at all time-points. This ranged from $47 \%$ in the unguided injection and physiotherapist-led exercise arm to $61 \%$ in the ultrasound-guided injection and physiotherapist-led exercise arm. However, it should be noted that the majority of those with missing values were missing just one EQ-5D score. There was an improvement in health outcomes over time, with scores obtained at 12-months higher than the baseline scores in all four arms. With respect to QALYs over the 12-month period, higher scores were obtained in the physiotherapist-led exercise and ultrasound-guided injection 
groups when compared to the advice and exercise leaflet and unguided injection groups, respectively. After adjusting for baseline utility, similar results were obtained.

\section{Cost-utility analysis}

The results from the cost-effectiveness analysis using the imputed dataset showed that physiotherapist-led exercise was more expensive (cost difference £155.99) and more effective (QALY difference 0.031) than the advice and exercise leaflet. The resulting ICER was $£ 5,031$ per QALY gained (Table 3) with an 85\% chance of physiotherapist-led exercise being costeffective at a cost-effectiveness threshold of $£ 20,000$ per QALY gained (Figures 1 and 2). Ultrasound-guided injection was also more expensive (cost difference £15.89) and more effective (QALY difference 0.024 ) than unguided injection. The resulting ICER was $£ 662$ per QALY gained (Table 3) with an $83 \%$ chance of ultrasound-guided injection being costeffective at a willingness to pay threshold of $£ 20,000$ per QALY gained (Figures 1 and 2).

\section{Time off work and Productivity Losses}

Participants in the advice and exercise leaflet and unguided injection groups took more time off work than those in the physiotherapist-led exercise and ultrasound-guided injection groups. Productivity losses in terms of lost wages were also higher in the advice and exercise leaflet and unguided injection groups. However, these differences were not statistically significant (Supplementary Table 4 and Table 1).

\section{Sensitivity Analysis}

From a UK NHS perspective, the results obtained were similar to the base case analysis i.e. physiotherapist-led exercise was cost-effective compared to providing an advice and exercise leaflet and ultrasound-guided injection was cost-effective compared to unguided injection (Table 3). Results from the 'within the table' analysis showed that a combination of ultrasoundguided injection and physiotherapist-led exercise was the most cost-effective intervention 
( $£ 6,225$ per QALY gained ), supporting findings from the base case analysis (Table 4). The results obtained when the EQ-5D-5L tariff [24] was used are similar to those obtained with the mapping algorithm [16] and support the finding that physiotherapist-led exercise is costeffective compared to the advice and exercise leaflet, and ultrasound-guided injection is costeffective when compared to unguided injection (Supplementary Tables 5 and 6).

\section{Discussion}

\section{Summary of the main findings}

This health economic evaluation showed that for the management of SAPS, physiotherapistled exercise was cost-effective compared to the advice and exercise leaflet and ultrasoundguided injection was cost-effective when compared to unguided injection. Sensitivity analysis using the within-the-table analysis yielded similar results to the at-the-margins approach indicating that the choice of method did not change the findings of the health economic analysis. Similar findings with respect to the choice of method have been obtained in previous studies [8]. A previous economic evaluation showed that a combination of injection and exercise was cost-effective compared to exercise alone [2]. The results from this study add to this evidence by showing that the approach to providing exercise (physiotherapist-led, individualised, progressed and supervised exercise rather than through a standardised advice and exercise leaflet) and injection (guided using ultrasound rather than unguided) is important in terms of increasing their cost-effectiveness.

The results of the trial on which the economic evaluation is based showed that physiotherapistled exercise was more effective than an exercise leaflet whilst ultrasound-guidance conferred no additional benefit over unguided corticosteroid injection [25]. The health economic results are similar in terms of the comparison between physiotherapist-led exercise and the advice and exercise leaflet, but different in terms of the comparison between ultrasound-guided and 
unguided injection. It is however important to note that the primary outcome of the clinical trial was the Shoulder Pain and Disability Index whilst that of the economic evaluation was total QALYs obtained from EQ-5D. Although the difference in QALYs obtained for both comparisons was very small and not statistically significant, the agreed approach in health economics is to conduct a cost-effectiveness analysis with costs and effects estimated jointly [26]. Whilst clinical research focuses on hypothesis testing, economic evaluation is aimed at estimation which may produce contradictory results as was seen in this study with the injection comparison [27]. An important issue which the results of this study raise relates to whether the use of generic quality of life measures such as the EQ-5D are sufficient for assessing the costeffectiveness of interventions in this clinical syndrome. Previous research has shown that generic quality of life measures may be insensitive to changes in some disease areas including musculoskeletal conditions such as osteoarthritis (OA) [28-30].

\section{Strengths and weaknesses of the study}

A strength of the study is the factorial nature of the trial, which enabled us to compare four interventions within the same analysis.

A possible limitation of the economic analysis is the fact that the cost of training clinicians to perform ultrasound-guided injection was not included in the analysis and it could be argued that this may have under-estimated the cost of the ultrasound-guided injection. The cost and time required to train clinical staff to perform ultrasound-guided injections is a potential barrier to services. However, it should be noted that there are a number of difficulties associated with the estimation of a per patient training cost within economic evaluation studies and also the training received by clinicians would be used for a large number of patients over a number of years which would result in a low mean cost per patient. A further limitation was that only $76 \%$ of participants had complete data at all time-points, however, the missing data were accounted for using multiple imputations. 


\title{
Meaning of the results
}

This health economic analysis has shown that physiotherapist-led exercise and ultrasoundguided injection are more cost-effective treatment options for SAPS than an advice and exercise leaflet or unguided shoulder injection respectively. However, this conclusion should be interpreted in the light of the clinical results which showed that physiotherapist-led exercise conferred superior benefits for patients compared to the advice and leaflet yet ultrasound guided injection conferred no additional benefit over unguided injection [25].

\begin{abstract}
Acknowledgements: This project was undertaken with the support of Keele Clinical Trials Unit, Keele University, UK. The authors would like to thank Kris Clarkson, Treena Larkin, and Sue Weir. We would also like to thank the clinicians, managerial and administrative staff of the Midlands Partnership NHS Foundation Trust, and members of the trial steering committee and data monitoring committee.
\end{abstract}

Funding: This paper presents independent research funded by the National Institute for Health Research (NIHR) under its Research for Patient Benefit programme (Grant Reference Number PB-PG-1207-15064) and the Arthritis Research UK Primary Care Centre grant (Grant Number 18139). NEF and EMH are both NIHR Senior Investigators. NEF was supported through an NIHR Research Professorship (NIHR-RP- 011-015). The views expressed are those of the authors and not necessarily those of the NHS, the NIHR or the Department of Health and Social Care.

Conflicts of interest: None to declare 


\section{References}

1. Urwin M, Symmons D, Allison T et al. Estimating the burden of musculoskeletal disorders in the community: the comparative prevalence of symptoms at different anatomical sites, and the relation to social deprivation. Ann Rheum Dis 1998;57:649-55.

2. Jowett S, Crawshaw DP, Helliwell PS, Hensor EM, Hay EM, Conaghan PG. Costeffectiveness of exercise therapy after corticosteroid injection for moderate to severe shoulder pain due to subacromial impingement syndrome: a trial-based analysis. Rheumatology. 2013;52:1485-91.

3. Luime JJ, Koes BW, Hendriksen IJ, Burdorf A, Verhagen AP, Miedema HS, Verhaar JA. Prevalence and incidence of shoulder pain in the general population; a systematic review. Scand J Rheumatol. 2004;33:73-81.

4. Van der Windt DA, Koes BW, de Jong BA, Bouter LM. Shoulder disorders in general practice: incidence, patient characteristics, and management. Annals of the rheumatic diseases. Ann Rheum Dis 1995;54:959-64.

5. Hanchard N, Cummins J, Jeffries J. Evidence-Based Clinical Guidelines for the Diagnosis, Assessment and Physiotherapy Management of Shoulder Impingement Syndrome. London: Chartered Society of Physiotherapy, 2004.

6. Kulkarni R, Gibson J, Brownson P, Thomas M, Rangan A, Carr AJ, et al. Subacromial shoulder pain. Shoulder \& Elbow. 2015;7:135-43.

7. Roddy E, Zwierska I, Hay EM, Jowett S, Lewis M, Stevenson K, et al. Subacromial impingement syndrome and pain: protocol for a randomised controlled trial of exercise and corticosteroid injection (the SUPPORT trial). BMC Musculoskelet Disord. 2014;15:81.

8. Dakin H, Gray A 2017 Economic evaluation of factorial randomised controlled trials: challenges, methods and recommendations Stat Med 2017;36:2814-2830

9. Oppong R, Jowett S, Nicholls E, Whitehurst DG, Hill S, Hammond A, et al. Joint protection and hand exercises for hand osteoarthritis: an economic evaluation comparing methods for the analysis of factorial trials. Rheumatology. 2014;54:876-83.

10. Curtis L. Unit Costs of Health and Social Care 2012 PSSRU. University of Kent: Canterbury. 2012.

11. BMJ Group. British National Formulary (BNF) 66. London: BMJ Group and RPS Publishing . 2012.

12. NHS reference cost 2012/2013 https://www.gov.uk/government/publications/nhsreference-costs-2012-to-2013 (accessed 16/07/2018)

13. Stevenson, K., Jackson, S., Shufflebotham, J., Roddy, E. and Foster, N.E., Development and delivery of a physiotherapist-led exercise intervention in a randomised controlled trial for subacromial impingement syndrome (the SUPPORT trial). Physiotherapy, 2017;103:379-386. 
14. Herdman M, Gudex C, Lloyd A, Janssen MF, Kind P, Parkin D, et al. Development and preliminary testing of the new five-level version of EQ-5D (EQ-5D-5L). Qual Life Res. 2011;20:1727-36.

15. NICE: Position statement on use of the EQ-5D-5L valuation set 2017 https://www.nice.org.uk/about/what-we-do/our-programmes/nice-guidance/technologyappraisal-guidance/eq-5d-51 (accessed 20/02/2019)

16. Van Hout B, Janssen MF, Feng YS, Kohlmann T, Busschbach J, Golicki D, et al. Interim scoring for the EQ-5D-5L: mapping the EQ-5D-5L to EQ-5D-3L value sets. Value in health. 2012 Jul 1;15(5):708-15.

17. Rubin DB. Multiple imputation for nonresponse in surveys. New York, USA: John Willey \& Sons 1987.

18. White IR, Royston P, Wood AM. Multiple imputation using chained equations: Issues and guidance for practice Stat Med 2011; 30: 337-99.

19. Matthews J, Altman DG, Campbell MJ, Royston P. Analysis of serial measurements in medical research. BMJ 1990;300:230-5.

20. Manca A, Hawkins N, Sculpher MJ. Estimating mean QALYs in trial based cost effectiveness analysis: the importance of controlling for baseline utility. Health Econ. 2005;14:487-496.

21. Van Hout BA, Al MJ, Gordon GS, Rutten FF. Costs, effects and C/E-ratios alongside a clinical trial. Health Econ 1994;3:309-19.

22. STATA 2012 StataCorp. LP. Stata/IC 12.1 for Windows. 2012

23. Standard Occupational Classification (SOC) 2000 http://www.ons.gov.uk/ons/guidemethod/classifications/archived-standard-classifications/standard-occupational-classification2000/about-soc-2000/index.html

24. Devlin NJ, Shah KK, Feng Y, Mulhern B, van Hout B. Valuing health-related quality of life: An EQ-5 D-5 L value set for E ngland. Health economics. 2018 Jan;27(1):7-22.

25. Roddy E, Ogollah R, Oppong R, Zwierska I, Datta P, Hall A et al. Optimising the outcome of exercise and corticosteroid injection in patients with subacromial impingement syndrome: a factorial randomised trial Br J Sports Med http://dx.doi.org/10.1136/bjsports2019-101268

26. Dakin H, Wordsworth S. Cost-Minimisation Analysis Versus Cost-Effectiveness Analysis, Revisited. Health Econ 2013;22:22-34

27. Whitehurst DGT, Bryan S Trial-based and economic analyses: the unhelpful quest for conformity. Trials 2013;14:421 
28. National Institute for Health and Care Excellence. Guide to the Methods of Technology Appraisal. London, UK: National Institute for Health and Care Excellence, 2013.

29. Brazier JE, Harper R, Munro J, Walters SJ, Snaith ML. Generic and condition-specific outcome measures for people with osteoarthritis of the knee. Rheumatology 1999;38:870-7.

30. Oppong R, Jowett S, Nicholls E, Whitehurst DG, Hill S, Hammond A, et al. Joint protection and hand exercises for hand osteoarthritis: an economic evaluation comparing methods for the analysis of factorial trials. Rheumatology. 2014;54:876-83. 
Table 1: Mean health care cost (SD) per patient over 12 months (complete cases) ( $£, 2019$ prices)

\begin{tabular}{|c|c|c|c|c|c|c|}
\hline & \multicolumn{3}{|c|}{ Injection intervention group } & \multicolumn{3}{|c|}{ Exercise intervention group } \\
\hline & $\begin{array}{l}\text { US-guided } \\
\quad(n=95)\end{array}$ & $\begin{array}{c}\text { Unguided } \\
(\mathrm{n}=99)\end{array}$ & $\begin{array}{c}\text { Mean difference } \\
(95 \% \mathrm{CI})\end{array}$ & $\begin{array}{c}\begin{array}{c}\text { Physiotherapist- } \\
\text { led } \\
(\mathrm{n}=95)\end{array} \\
\end{array}$ & $\begin{array}{c}\begin{array}{c}\text { Advice and Exercise } \\
\text { Leaflet } \\
(\mathbf{n}=99)\end{array} \\
\end{array}$ & $\begin{array}{c}\text { Mean difference } \\
(95 \% \text { CI })\end{array}$ \\
\hline Intervention $\operatorname{cost} \mathrm{t}^{\mathrm{a}}$ & $\begin{array}{l}186.01 \\
(126.50)\end{array}$ & $\begin{array}{l}107.12 \\
(127.33)\end{array}$ & $\begin{array}{c}78.89 \\
(41.98,113.59)\end{array}$ & $258.92(96.73)$ & $37.15(36.58)$ & $\begin{array}{c}221.77 \\
(196.45,241.00)\end{array}$ \\
\hline $\begin{array}{l}\text { PRIMARY CARE } \\
\text { CONTACTS }\end{array}$ & & & & & & \\
\hline General Practitioner & $39.16(75.27)$ & $43.30(97.90)$ & $\begin{array}{c}-4.14 \\
(-28.39,20.59)\end{array}$ & $28.01(70.66)$ & $53.99(99.52)$ & $\begin{array}{c}-25.98 \\
(-49.65,-3.08)\end{array}$ \\
\hline Practice Nurse & $1.28(11.11)$ & $0.58(4.16)$ & $\begin{array}{c}0.70 \\
(-0.84,3.99)\end{array}$ & $1.21(9.73)$ & $0.92(6.73)$ & $\begin{array}{c}0.29 \\
(-1.69,3.46)\end{array}$ \\
\hline $\begin{array}{l}\text { Other professionals in } \\
\text { GP practice } \\
\text { SECONDARY CARE } \\
\text { CONTACTS }\end{array}$ & $14.48(40.98)$ & $22.58(59.73)$ & $\begin{array}{c}-8.10 \\
(-22.99,4.19)\end{array}$ & $24.52(58.25)$ & $12.94(43.47)$ & $\begin{array}{c}11.58 \\
(-2.64,26.78)\end{array}$ \\
\hline Physiotherapist ${ }^{b}$ & $37.27(86.59)$ & $32.69(86.02)$ & $\begin{array}{c}4.58 \\
(-20.45,27.03)\end{array}$ & $53.31(102.03)$ & $17.30(63.03)$ & $\begin{array}{c}36.01 \\
(14.52,62.41)\end{array}$ \\
\hline NHS consultants & $26.97(60.56)$ & $49.08(93.36)$ & $\begin{array}{c}-22.11 \\
(-43.75,-1.21)\end{array}$ & $38.15(74.24)$ & $38.34(76.12)$ & $\begin{array}{c}-0.19 \\
(-23.44,21.53)\end{array}$ \\
\hline $\begin{array}{l}\text { Private consultants and } \\
\text { Physiotherapists }\end{array}$ & $18.07(83.84)$ & $8.98(35.80)$ & $\begin{array}{c}9.09 \\
(-5.96,30.28)\end{array}$ & $6.17(27.97)$ & $20.40(84.94)$ & $\begin{array}{c}-14.23 \\
(-38.09,-0.79)\end{array}$ \\
\hline $\begin{array}{l}\text { Other professional in } \\
\text { NHS hospitals } \\
\text { REPEAT INJECTIONS }\end{array}$ & $1.58(11.87)$ & $2.07(11.14)$ & $\begin{array}{c}-0.49 \\
(-3.61,2.93)\end{array}$ & $1.46(11.81)$ & $2.20(11.19)$ & $\begin{array}{c}-0.74 \\
(-3.82,2.81)\end{array}$ \\
\hline Repeat Injections in NHS & $35.96(98.07)$ & $51.07(118.07)$ & $\begin{array}{c}-15.11 \\
(-45.35,17.64)\end{array}$ & $33.08(104.93)$ & $53.83(111.82)$ & $\begin{array}{c}-20.75 \\
(-50.81,8.58)\end{array}$ \\
\hline $\begin{array}{l}\text { Repeat Injections in } \\
\text { private practice } \\
\text { DIAGNOSTIC TESTS }\end{array}$ & $1.43(14.02)$ & - & $\begin{array}{c}1.43 \\
(0,5.88)\end{array}$ & - & $1.38(13.73)$ & $\begin{array}{c}-1.38 \\
(-5.11,0)\end{array}$ \\
\hline Diagnostic tests in NHS & $36.27(105.24)$ & $72.97(144.77)$ & $\begin{array}{c}-36.70 \\
(-70.06,-5.69)\end{array}$ & $45.44(114.17)$ & $64.18(139.89)$ & $\begin{array}{c}-18.74 \\
(-53.69,17.34)\end{array}$ \\
\hline $\begin{array}{l}\text { Diagnostic tests in private } \\
\text { practice }\end{array}$ & - & $1.76(12.98)$ & $\begin{array}{c}-1.76 \\
(-5.38,0)\end{array}$ & - & $1.76(12.98)$ & $\begin{array}{c}-1.76 \\
(-4.96,0)\end{array}$ \\
\hline
\end{tabular}




\section{MEDICATION}

Prescribed medication

$\begin{array}{lcc}5.70(16.62) & 7.80(16.96) & -2.10 \\ & & (-6.74,2.51) \\ 10.15(20.01) & 18.86(57.93) & -8.71\end{array}$

$3.71(7.19)$

$9.71(22.08)$

$-6.00$

Over the counter medication

$10.15(20.01) \quad 18.86(57.93)$

$(-22,80,1.11)$

$13.85(45.62)$

$15.31(42.19)$

$(-10.86,-1.78)$

$-1.46$

\section{WORK RELATED \\ OUTCOMES}

Productivity costs

388.06

823.56

$-435.50$

$562.54(2713.56) \quad 656.13(3317.80)$

$(-12.94,11.17)$

\section{TOTAL COSTS}

(1638.82)

$(3924.91)$
$(-1472.23,239.61)$

$4.70,654.54)$

Total health care $\operatorname{costs}^{\mathrm{c}}(\mathrm{n}=256)$

428.01

412.12

15.89

$498.06(326.05) \quad 342.07(346.11)$

155.99

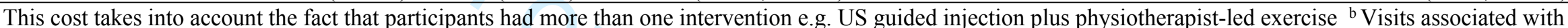
the intervention have been excluded ${ }^{\mathrm{c}}$ Costs have been imputed 
Table 2: Mean health outcomes (SD) over 12 months (imputed data)

\begin{tabular}{|c|c|c|c|c|c|c|}
\hline & \multicolumn{3}{|c|}{ Injection intervention group } & \multicolumn{3}{|c|}{ Exercise intervention group } \\
\hline & $\begin{array}{l}\text { US-guided } \\
(n=128)\end{array}$ & $\begin{array}{l}\text { Unguided } \\
(n=128)\end{array}$ & $\begin{array}{c}\text { Mean difference } \\
(95 \% \mathrm{CI})\end{array}$ & $\begin{array}{l}\text { Physiotherapist-led } \\
(n=128)\end{array}$ & $\begin{array}{c}\text { Advice \& exercise leaflet } \\
(\mathrm{n}=128)\end{array}$ & $\begin{array}{l}\text { Mean difference } \\
(95 \% \mathrm{CI})\end{array}$ \\
\hline \multicolumn{7}{|l|}{ EQ-5D scores } \\
\hline Baseline & $0.494(0.255)$ & $0.501(0.235)$ & $-0.007(-0.065,0.052)$ & $0.504(0.243)$ & $0.492(0.247)$ & $0.012(-0.042,0.078)$ \\
\hline 6 weeks & $0.599(0.240)$ & $0.574(0.236)$ & $0.025(0.031,0.080)$ & $0.601(0.242)$ & $0.572(0.234)$ & $0.029(-0.025,0.087)$ \\
\hline 6 months & $0.577(0.245)$ & $0.549(0.261)$ & $0.028(-0.031,0.088)$ & $0.584(0.246)$ & $0.541(0.259)$ & $0.043(-0.018,0.103)$ \\
\hline 12 months & $0.599(0.248)$ & $0.594(0.271)$ & $0.005(-0.058,0.066)$ & $0.606(0.268)$ & $0.588(0.252)$ & $0.018(-0.052,0.081)$ \\
\hline Total QALYs & $0.584(0.225)$ & $0.564(0.230)$ & $0.020(-0.035,0.072)$ & $0.594(0.227)$ & $0.555(0.226)$ & $0.039(-0.013,0.097)$ \\
\hline $\begin{array}{l}\text { Total adjusted } \\
\text { QALYs a }\end{array}$ & 0.586 & 0.562 & $0.024(-0.018,0.066)$ & 0.589 & 0.558 & $0.031(-0.010,0.073)$ \\
\hline
\end{tabular}

${ }^{\mathrm{a}} \mathrm{QALYs}$ adjusted for baseline utility 
Table 3: Cost-utility analysis (imputed data)

\begin{tabular}{|c|c|c|c|c|c|c|}
\hline & \multicolumn{2}{|c|}{ Injection intervention group } & & \multicolumn{3}{|c|}{ Exercise intervention group } \\
\hline & $\begin{array}{l}\text { US-guided } \\
(n=128)\end{array}$ & $\begin{array}{l}\text { Unguided } \\
(n=128)\end{array}$ & & $\begin{array}{l}\text { Physiotherapist-led } \\
(\mathrm{n}=128)\end{array}$ & $\begin{array}{c}\begin{array}{c}\text { Advice \& Exercise } \\
\text { Leaflet } \\
(\mathbf{n}=\mathbf{1 2 8})\end{array} \\
\end{array}$ & \\
\hline & Mean & Mean & $\begin{array}{l}\text { Mean difference } \\
\text { (Confidence } \\
\text { Interval) }\end{array}$ & Mean & Mean & $\begin{array}{l}\text { Mean difference } \\
\text { (Confidence } \\
\text { Interval) }\end{array}$ \\
\hline \multicolumn{7}{|c|}{ HEALTHCARE PERSPECTIVE } \\
\hline Cost & $£ 428.01$ & $£ 412.12$ & $\begin{array}{c}£ 15.89 \\
(-59.36,109.86)\end{array}$ & $£ 498.06$ & $£ 342.07$ & $\begin{array}{c}£ 155.99 \\
(69.02,241.93)\end{array}$ \\
\hline QALYs ${ }^{a}$ & 0.586 & 0.562 & $\begin{array}{c}0.024 \\
(-0.018,0.066)\end{array}$ & 0.589 & 0.558 & $\begin{array}{c}0.031 \\
(-0.010,0.073)\end{array}$ \\
\hline ICER & $£ 662$ per QALY gained & & & $£ 5,031$ per QALY gained & & \\
\hline \multicolumn{7}{|c|}{ NHS PERSPECTIVE } \\
\hline Cost & $£ 398.25$ & $£ 382.04$ & $\begin{array}{c}£ 15.85 \\
(-64.32,90.22)\end{array}$ & $£ 476.02$ & $£ 304.27$ & $\begin{array}{c}£ 171.75 \\
(98.44,251.88)\end{array}$ \\
\hline QALYs ${ }^{a}$ & 0.586 & 0.562 & $\begin{array}{c}0.024 \\
(-0.018,0.066)\end{array}$ & 0.589 & 0.558 & $\begin{array}{c}0.031 \\
(-0.010,0.073)\end{array}$ \\
\hline ICER & $£ 660$ per QALY gained & & & $£ 5,540$ per QALY gained & & \\
\hline
\end{tabular}


Table 4: Cost-utility analysis "within the table" analysis

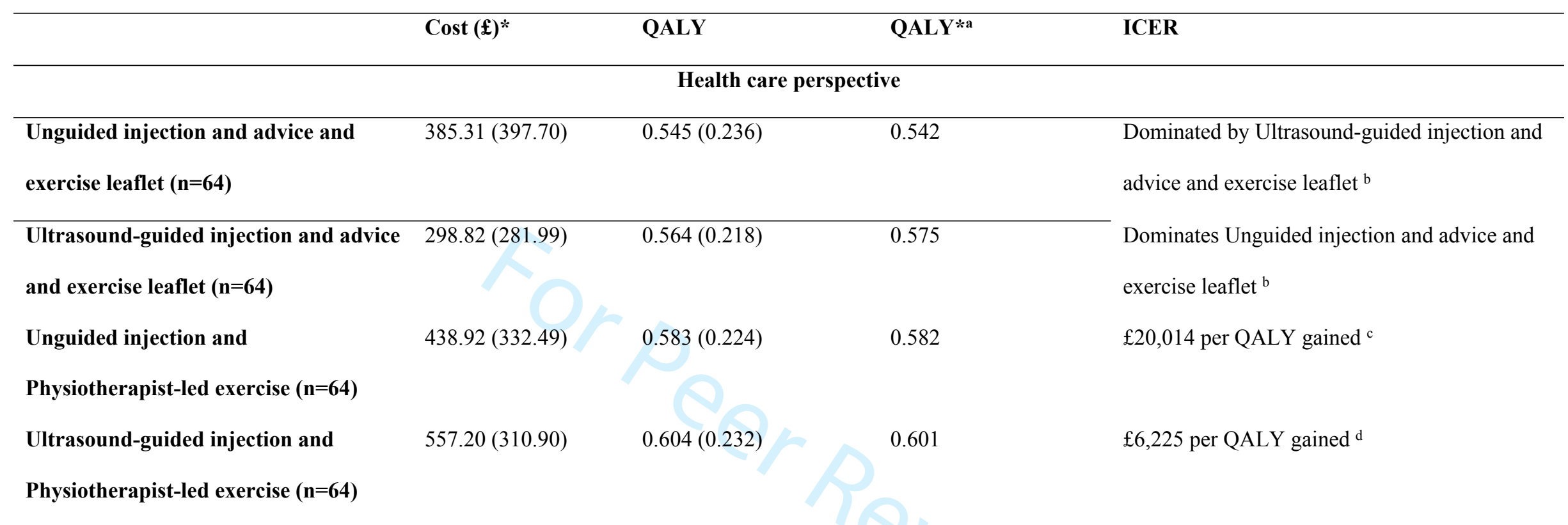

NHS perspective

\begin{tabular}{|c|c|c|c|c|}
\hline & Cost* & QALY & QALY*a & ICER \\
\hline Unguided injection and advice and & $347.87(385.17)$ & $0.545(0.236)$ & 0.542 & Dominated by Ultrasound-guided injection and \\
\hline exercise leaflet $(n=64)$ & & & & advice and exercise leaflet ${ }^{b}$ \\
\hline Ultrasound-guided injection and advice & $260.66(220.67)$ & $0.564(0.218)$ & 0.575 & Dominates Unguided injection and advice and \\
\hline and exercise leaflet $(n=64)$ & & & & exercise leaflet $^{b}$ \\
\hline Unguided injection and & $416.21(324.81)$ & $0.583(0.224)$ & 0.582 & $£ 22,221$ per QALY gained ${ }^{\mathrm{c}}$ \\
\hline
\end{tabular}

Physiotherapist-led exercise $(n=64)$ 


\section{Ultrasound-guided injection and}

$535.83(305.61)$

$0.604(0.232)$

0.601

$£ 6,295$ per QALY gained ${ }^{\mathrm{d}}$

Physiotherapist-led exercise $(n=64)$

*Cost and QALYs imputed a Adjusted for baseline utility ${ }^{\mathrm{b}}$ Ultrasound-guided injection and advice and exercise leaflet versus Unguided injection and advice and exercise leaflet ${ }^{\mathrm{c}}$ Unguided injection and Physiotherapist-led exercise versus Ultrasound-guided injection and advice and exercise leaflet ${ }^{\mathrm{d}}$ Ultrasound-guided injection and

Physiotherapist-led exercise versus Unguided injection and Physiotherapist-led exercise 
Figure 1: Cost-effectiveness plane

\section{Cost-effectiveness plane}
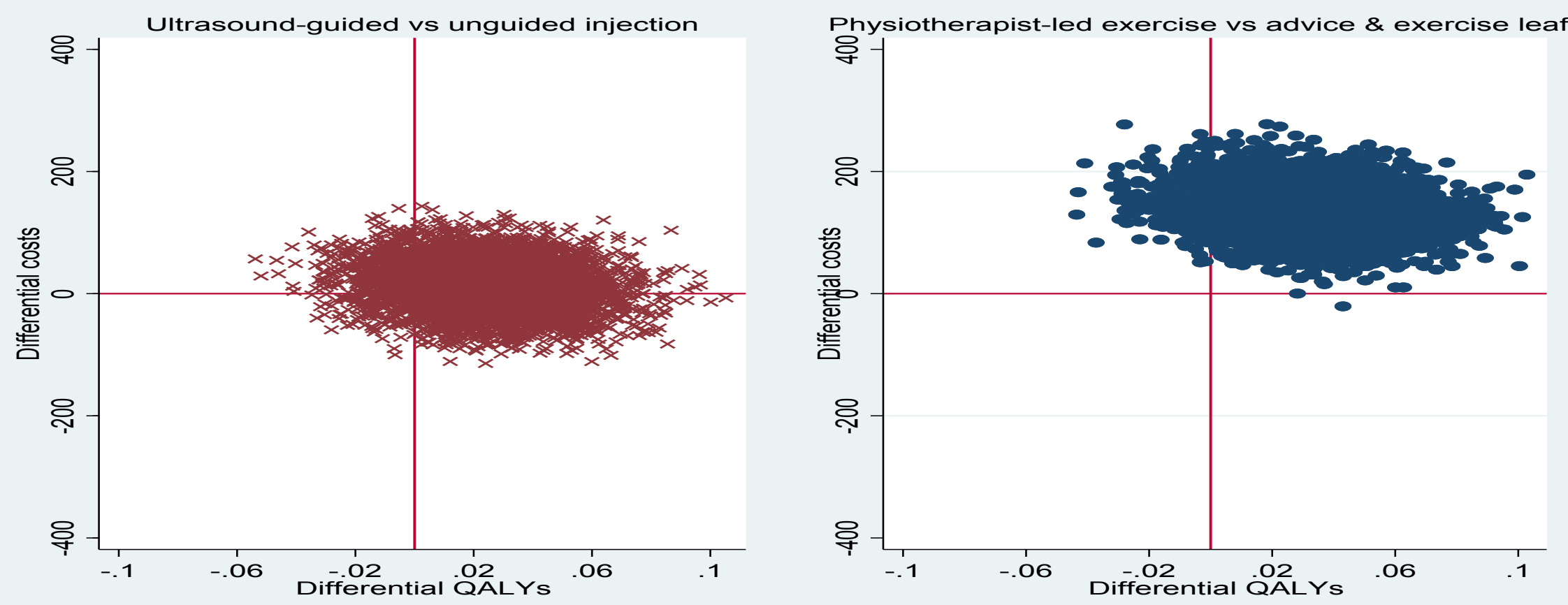
Figure 2: Cost-effectiveness acceptability curve

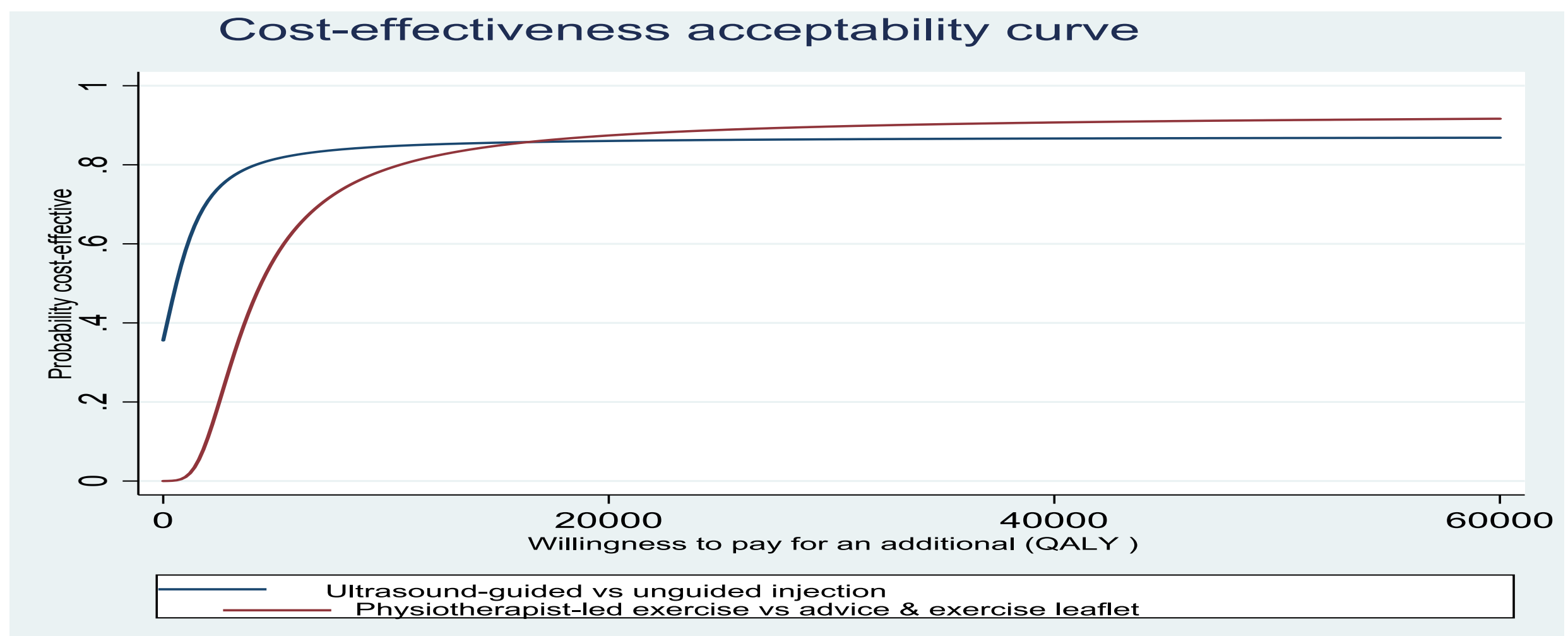


Supplementary Table 1: Unit cost of health care resource use

\begin{tabular}{|c|c|}
\hline \multirow{2}{*}{\multicolumn{2}{|c|}{$\begin{array}{l}\text { Health care resource } \\
\text { Primary care contacts }\end{array}$}} \\
\hline & \\
\hline GP at practice & 43 \\
\hline GP at home & 53.58 \\
\hline Nurse at practice & 10.30 \\
\hline Nurse at home & 23.30 \\
\hline \multicolumn{2}{|l|}{ Secondary care contacts } \\
\hline Physiotherapist visits & 34 \\
\hline Consultants & 52 \\
\hline Specialists A\&E visits & 62 \\
\hline \multicolumn{2}{|l|}{ Intervention $\cos ^{t^{\mathrm{a}}}$} \\
\hline Physiotherapist-led exercise & 34 per visit $^{b}$ \\
\hline Advice and exercise leaflet & $1^{\mathrm{c}}$ \\
\hline Ultrasound-guided injection & $65^{\mathrm{d}}$ \\
\hline Unguided injection & $0^{\mathrm{e}}$ \\
\hline Prescribed Medication & Participant specific \\
\hline Over the counter medication & Participant specific \\
\hline Medical investigations/Interventions & Participant specific \\
\hline
\end{tabular}

${ }^{a}$ These costs are what was actually used for the analysis ${ }^{b}$ This value was multiplied by the number of times a participant saw a physiotherapist. Thus, the cost varied from participant to participant ${ }^{\mathrm{c}}$ This is the cost of the leaflet ${ }^{\mathrm{d}}$ Includes cost of the ultrasound procedure ${ }^{\mathrm{e}}$ Assumed to be zero since all patients had a similar injection and the only difference between the ultrasound-guided and unguided injection was the cost of the ultrasound. 
Supplementary Table 2: Mean (SD) NHS resource use per patient (complete cases)

\begin{tabular}{|c|c|c|c|c|c|c|}
\hline \multicolumn{4}{|c|}{ Injection intervention group } & \multicolumn{3}{|c|}{ Exercise intervention group } \\
\hline & US-guided (n=95) & $\begin{array}{c}\text { Unguided } \\
(\mathrm{n}=99)\end{array}$ & $\begin{array}{l}\text { Difference } \\
\text { (Confidence } \\
\text { Interval) } \\
\end{array}$ & $\begin{array}{l}\text { Physiotherapist-led } \\
\qquad(\mathrm{n}=95)\end{array}$ & $\begin{array}{l}\text { Advice and Exercise } \\
\text { Leaflet }(n=99)\end{array}$ & $\begin{array}{l}\text { Difference } \\
\text { (Confidence } \\
\text { Interval) } \\
\end{array}$ \\
\hline \multicolumn{7}{|c|}{ PRIMARY CARE CONTACTS } \\
\hline GP (surgery) & $0.80(1.51)$ & $0.90(2.03)$ & $\begin{array}{c}-0.10 \\
(-0.62,0.38)\end{array}$ & $0.57(1.41)$ & $1.12(2.07)$ & $\begin{array}{c}-\mathbf{0 . 5 5} \\
(-1.07,-0.07)\end{array}$ \\
\hline GP (home visit) & $0.01(0.10)$ & 0 & $\begin{array}{c}0.01 \\
(0.00,0.04)\end{array}$ & $0.01(0.10)$ & 0 & $\begin{array}{c}0.01 \\
(0.00,0.04)\end{array}$ \\
\hline Nurse visits (surgery) & $0.14(0.96)$ & $0.05(0.36)$ & $\begin{array}{c}0.09 \\
(-0.08,0.35)\end{array}$ & $0.11(0.84)$ & $0.08(0.58)$ & $\begin{array}{c}0.03 \\
(-0.14,0.28)\end{array}$ \\
\hline Nurse (home visit) & 0 & 0 & 0 & 0 & 0 & $\mathbf{0}$ \\
\hline $\begin{array}{r}\text { Other professionals } \\
\text { attached to GP practice } \\
\text { (surgery) }\end{array}$ & $0.35(0.91)$ & $0.48(1.31)$ & $\begin{array}{c}-0.13 \\
(-0.46,0.17)\end{array}$ & $0.52(1.22)$ & $0.32(1.04)$ & $\begin{array}{c}0.20 \\
(-0.13,0.50)\end{array}$ \\
\hline $\begin{array}{r}{ }^{\mathrm{a}} \text { Other professionals } \\
\text { attached to GP practice } \\
\text { (home) }\end{array}$ & $0.04(0.29)$ & $0.08(0.80)$ & $\begin{array}{c}-0.04 \\
(-0.26,0.08)\end{array}$ & $0.13(0.87)$ & 0 & $\begin{array}{c}0.13 \\
(0.02,0.38)\end{array}$ \\
\hline \multicolumn{7}{|c|}{ SECONDARY CARE CONTACTS } \\
\hline Consultant & $0.46(1.04)$ & $0.75(1.48)$ & $\begin{array}{c}-0.29 \\
(-0.64,0.08)\end{array}$ & $0.60(1.27)$ & $0.62(1.31)$ & $\begin{array}{c}-0.02 \\
(-0.40,0.35)\end{array}$ \\
\hline Physiotherapist ${ }^{b}$ & $2.79(3.96)$ & $2.45(3.78)$ & $\begin{array}{c}0.34 \\
(-0.67,1.47)\end{array}$ & $4.87(4.21)$ & $0.45(1.66)$ & $\begin{array}{c}4.42 \\
(3.51,5.35)\end{array}$ \\
\hline Specialist A\&E & 0 & $0.06(0.42)$ & $\begin{array}{c}-0.06 \\
(-0.19,-0.01)\end{array}$ & $0.04(0.41)$ & $0.02(0.14)$ & $\begin{array}{c}0.02 \\
(-0.04,0.12)\end{array}$ \\
\hline Hospital Nurse & 0 & $0.08(0.34)$ & $\begin{array}{c}-0.08 \\
(-0.17,-0.03)\end{array}$ & $0.02(0.14)$ & $0.06(0.31)$ & $\begin{array}{c}0.04 \\
(-0.12,0.02)\end{array}$ \\
\hline Acupuncturist & 0 & $0.01(0.10)$ & $\begin{array}{c}-0.01 \\
(-0.03,0)\end{array}$ & 0 & $0.01(0.10)$ & $\begin{array}{c}-0.01 \\
(-0.03,0)\end{array}$ \\
\hline${ }^{\mathrm{c}}$ Other professionals & $0.06(0.35)$ & $0.06(0.31)$ & $\begin{array}{c}0 \\
(-0.08,0.10)\end{array}$ & $0.04(0.32)$ & $0.08(0.34)$ & $\begin{array}{c}-0.04 \\
(-0.13,0.07)\end{array}$ \\
\hline Repeat Injections & $0.26(0.72)$ & $0.37(0.86)$ & $\begin{array}{c}-0.11 \\
(-0.28,0.07)\end{array}$ & $0.24(0.77)$ & $0.39(0.82)$ & $\begin{array}{c}-0.15 \\
(-0.30,0.05)\end{array}$ \\
\hline
\end{tabular}

${ }^{\mathrm{a}}$ This includes contacts with professionals such as work nurse sister and consultant ${ }^{\mathrm{b}}$ Physiotherapy visits that were part of the intervention have been included here ${ }^{\mathrm{c}}$ Includes contacts with professionals such as work nurse sister, sports therapist, massage masseur and kinesiologist 
Supplementary Table 3: Mean (SD) prescribed drugs and medical investigations per patient over 12 months (complete cases)

\begin{tabular}{|c|c|c|c|c|c|c|}
\hline \multicolumn{4}{|c|}{ Injection intervention group } & \multicolumn{3}{|c|}{ Exercise intervention group } \\
\hline & US-guided $(n=95)$ & Unguided (n=99) & $\begin{array}{c}\text { Difference } \\
\text { (Confidence } \\
\text { Interval) } \\
\end{array}$ & $\begin{array}{l}\text { Physiotherapist-led } \\
(n=95)\end{array}$ & $\begin{array}{l}\text { Advice and Exercise } \\
\text { Leaflet }(n=99)\end{array}$ & $\begin{array}{c}\text { Difference } \\
\text { (Confidence } \\
\text { Interval) } \\
\end{array}$ \\
\hline \multicolumn{7}{|c|}{ PRESCRIBED DRUGS } \\
\hline Paracetamol & $0.38(1.45)$ & $0.52(2.00)$ & $\begin{array}{c}-0.14 \\
(-0.62,0.32)\end{array}$ & $0.37(1.39)$ & $0.53(2.04)$ & $\begin{array}{c}-0.16 \\
(-0.72,0.27)\end{array}$ \\
\hline Ibuprofen & $0.31(1.38)$ & $0.75(2.89)$ & $\begin{array}{c}-0.44 \\
(-1.14,0.08)\end{array}$ & $0.28(1.29)$ & $0.78(2.92)$ & $\begin{array}{c}-0.5 \\
(-1.24,0.06)\end{array}$ \\
\hline Co-codamol & $0.77(2.11)$ & $1.19(2.71)$ & $\begin{array}{c}-0.42 \\
(-1.13,0.24)\end{array}$ & $1(2.46)$ & $0.97(2.43)$ & $\begin{array}{c}0.03 \\
(-0.64,0.77)\end{array}$ \\
\hline Diclofenac & $0.04(0.25)$ & $0.09(0.81)$ & $\begin{array}{c}-0.05 \\
(-0.28,0.06) \\
\end{array}$ & $0.01(0.10)$ & $0.12(0.84)$ & $\begin{array}{c}-0.11 \\
(-0.34,-0.001)\end{array}$ \\
\hline \multicolumn{7}{|c|}{ MEDICAL INVESTIGATIONS } \\
\hline Blood test & $0.09(0.41)$ & $0.26(0.79)$ & $\begin{array}{c}-\mathbf{0 . 1 7} \\
(-0.04,-0.03)\end{array}$ & $0.05(0.22)$ & $0.30(0.85)$ & $\begin{array}{c}-0.25 \\
(-0.46,-0.10)\end{array}$ \\
\hline X-ray & $0.13(0.39)$ & $0.29(0.72)$ & $\begin{array}{c}-0.16 \\
(-0.33,-0.16)\end{array}$ & $0.16(0.55)$ & $0.26(0.62)$ & $\begin{array}{c}-0.10 \\
(-0.25,0.06)\end{array}$ \\
\hline Ultrasound & $0.11(0.35)$ & $0.33(0.73)$ & $\begin{array}{c}-0.22 \\
(0.39,-0.06)\end{array}$ & $0.17(0.54)$ & $0.28(0.62)$ & $\begin{array}{c}-0.11 \\
(-0.28,0.04)\end{array}$ \\
\hline MRI scan & $0.01(0.10)$ & $0.04(0.24)$ & $\begin{array}{c}-0.03 \\
(-0.10,0.01)\end{array}$ & $0.01(0.10)$ & $0.04(0.24)$ & $\begin{array}{c}-0.03 \\
(-0.10,0.01)\end{array}$ \\
\hline CT scan & 0 & $0.03(0.22)$ & $\begin{array}{c}-0.03 \\
(-0.10,0)\end{array}$ & 0 & $0.03(0.22)$ & $\begin{array}{c}-0.03 \\
(-0.10,0)\end{array}$ \\
\hline
\end{tabular}


Supplementary Table 4: Mean (SD) private resource use per patient over 12 months (complete cases)

\begin{tabular}{|c|c|c|c|c|c|c|}
\hline \multicolumn{4}{|c|}{ Injection intervention group } & \multicolumn{3}{|c|}{ Exercise intervention group } \\
\hline & US-guided (n=95) & $\begin{array}{c}\text { Unguided } \\
\text { (n=99) }\end{array}$ & $\begin{array}{l}\text { Difference } \\
\text { (Confidence } \\
\text { Interval) } \\
\end{array}$ & $\begin{array}{l}\text { Physiotherapist-led } \\
\qquad(\mathrm{n}=95)\end{array}$ & $\begin{array}{l}\text { Advice and Exercise } \\
\text { Leaflet }(\mathrm{n}=99)\end{array}$ & $\begin{array}{l}\text { Difference } \\
\text { (Confidence } \\
\text { Interval) } \\
\end{array}$ \\
\hline Consultant & $0.12(0.63)$ & $0.05(0.36)$ & $\begin{array}{c}0.07 \\
(-0.06,0.24)\end{array}$ & $0.03(0.33)$ & $0.13(0.65)$ & $\begin{array}{c}-0.1 \\
(-0.26,0.03)\end{array}$ \\
\hline Physiotherapist & $0.11(0.83)$ & $0.06(0.42)$ & $\begin{array}{c}0.05 \\
(-1.1,0.28)\end{array}$ & $0.07(0.44)$ & $0.09(0.81)$ & $\begin{array}{c}-0.02 \\
(-0.26,0.13)\end{array}$ \\
\hline Specialist & $0.02(0.21)$ & 0 & $\begin{array}{c}0.02 \\
(0,0.08)\end{array}$ & 0 & $0.02(0.20)$ & $\begin{array}{c}-0.02 \\
(-0.10,0)\end{array}$ \\
\hline Hospital Nurse & $0.01(0.10)$ & $0.01(0.10)$ & $\begin{array}{c}0 \\
(-0.02,0.03)\end{array}$ & $0.01(0.10)$ & $0.01(0.10)$ & $\begin{array}{c}0 \\
(-0.02,0.04)\end{array}$ \\
\hline Acupuncturist & $0.01(0.10)$ & 0 & $\begin{array}{c}0.01 \\
(0,0.04)\end{array}$ & 0 & $0.01(0.10)$ & $\begin{array}{c}-0.01 \\
(-0.04,0)\end{array}$ \\
\hline Osteopath & $0.07(0.72)$ & $0.06(0.60)$ & $\begin{array}{c}0.01 \\
(-0.13,0.27)\end{array}$ & 0 & $0.13(0.92)$ & $\begin{array}{c}-0.13 \\
(-0.35,0)\end{array}$ \\
\hline Other professionals & $0.08(0.58)$ & $0.06(0.45)$ & $\begin{array}{c}0.02 \\
(-0.12,0.17)\end{array}$ & $0.04(0.41)$ & $0.10(0.60)$ & $\begin{array}{c}-0.06 \\
(-0.24,0.08)\end{array}$ \\
\hline $\begin{array}{l}\text { Over the counter } \\
\text { medication }\end{array}$ & $2.86(5.52)$ & $6.22(22.15)$ & $\begin{array}{c}-3.36 \\
(-9.61,-0.09)\end{array}$ & $5.24(21.60)$ & $3.94(8.78)$ & $\begin{array}{c}1.30 \\
(-2.08,7.57)\end{array}$ \\
\hline Repeat Injection & $0.01(0.11)$ & 0 & $\begin{array}{c}0.01 \\
(0,0.04) \\
\end{array}$ & & $0.01(0.10)$ & $\begin{array}{c}-0.01 \\
(-0.04,0)\end{array}$ \\
\hline \multicolumn{7}{|c|}{ WORK RELATED ITEMS } \\
\hline $\begin{array}{l}\text { Time off work due to } \\
\text { shoulder problems } \\
\text { (mean days) }\end{array}$ & $3.49(14.63)$ & $8.70(44.50)$ & $\begin{array}{c}-1.68 \\
(-13.44,6.33)\end{array}$ & $5.29(24.00)$ & $6.97(40.57)$ & $\begin{array}{c}-5.21 \\
(-16.73,1.93)\end{array}$ \\
\hline
\end{tabular}


Supplementary Table 5: Cost-utility analysis 'at the margins' approach (with EQ-5D-5L tariff)

\begin{tabular}{|c|c|c|c|c|c|c|}
\hline & \multicolumn{2}{|c|}{ Injection intervention group } & & \multicolumn{3}{|c|}{ Exercise intervention group } \\
\hline & $\begin{array}{l}\text { US-guided } \\
(n=128)\end{array}$ & $\begin{array}{l}\text { Unguided } \\
(\mathrm{n}=128)\end{array}$ & & $\begin{array}{l}\text { Physiotherapist-led } \\
(\mathrm{n}=128)\end{array}$ & $\begin{array}{c}\text { Advice \& Exercise } \\
\text { Leaflet } \\
(\mathrm{n}=128)\end{array}$ & \\
\hline & Mean & Mean & $\begin{array}{l}\text { Mean difference } \\
\text { (Confidence } \\
\text { Interval) }\end{array}$ & Mean & Mean & $\begin{array}{l}\text { Mean difference } \\
\text { (Confidence } \\
\text { Interval) }\end{array}$ \\
\hline \multicolumn{7}{|c|}{ HEALTHCARE PERSPECTIVE } \\
\hline Cost & $£ 428.01$ & $£ 412.12$ & $\begin{array}{c}£ 15.89 \\
(-59.36,109.86)\end{array}$ & $£ 498.06$ & $£ 342.07$ & $\begin{array}{c}£ 155.99 \\
(69.02,241.93)\end{array}$ \\
\hline QALYs $^{\text {a }}$ & 0.697 & 0.667 & $\begin{array}{c}0.030 \\
(-0.009,0.070)\end{array}$ & 0.693 & 0.671 & $\begin{array}{c}0.023 \\
(-0.017,0.062)\end{array}$ \\
\hline ICER & $£ 529.7$ per QALY gained & & & $£ 6,782$ per QALY gained & & \\
\hline \multicolumn{7}{|c|}{ NHS PERSPECTIVE } \\
\hline Cost & $£ 398.25$ & $£ 382.04$ & $\begin{array}{c}£ 15.85 \\
(-64.32,90.22)\end{array}$ & $£ 476.02$ & $£ 304.27$ & $\begin{array}{c}£ 171.75 \\
(98.44,251.88)\end{array}$ \\
\hline QALYs a & 0.697 & 0.667 & $\begin{array}{c}0.030 \\
(-0.009,0.070)\end{array}$ & 0.693 & 0.671 & $\begin{array}{c}0.023 \\
(-0.017,0.062)\end{array}$ \\
\hline ICER & $£ 528.3$ per QALY gained & & & $£ 7,467$ per QALY gained & & \\
\hline
\end{tabular}

${ }^{\text {a }}$ Adjusted for baseline utility 
Supplementary Table 6: Cost-utility analysis "within the table" analysis (With EQ-5D-5L tariff)

\begin{tabular}{|c|c|c|c|}
\hline & Cost $(\mathfrak{f})^{*}$ & $\underline{\text { QALY*a }}$ & $\underline{\text { ICER }}$ \\
\hline & \multicolumn{3}{|c|}{ Health care perspective } \\
\hline Unguided injection and advice and exercise & $385.31(397.70)$ & $\underline{0.652}$ & N/A \\
\hline \multicolumn{4}{|l|}{ leaflet $(n=64)$} \\
\hline Unguided injection and Physiotherapist-led & $438.92(332.49)$ & $\underline{0.681}$ & $£ 1,848$ per QALY gained $b$ \\
\hline \multicolumn{4}{|l|}{ exercise $(n=64)$} \\
\hline Ultrasound-guided injection and advice and & $298.82(281.99)$ & 0.689 & Dominates Unguided injection and Physiotherapist-lec \\
\hline exercise leaflet $(n=64)$ & & & exercise $^{c}$ \\
\hline Ultrasound-guided injection and & $\underline{557.20(310.90)}$ & $\underline{0.705}$ & $£ 16,148$ per QALY gained ${ }^{\mathrm{d}}$ \\
\hline
\end{tabular}

\section{NHS perspective}

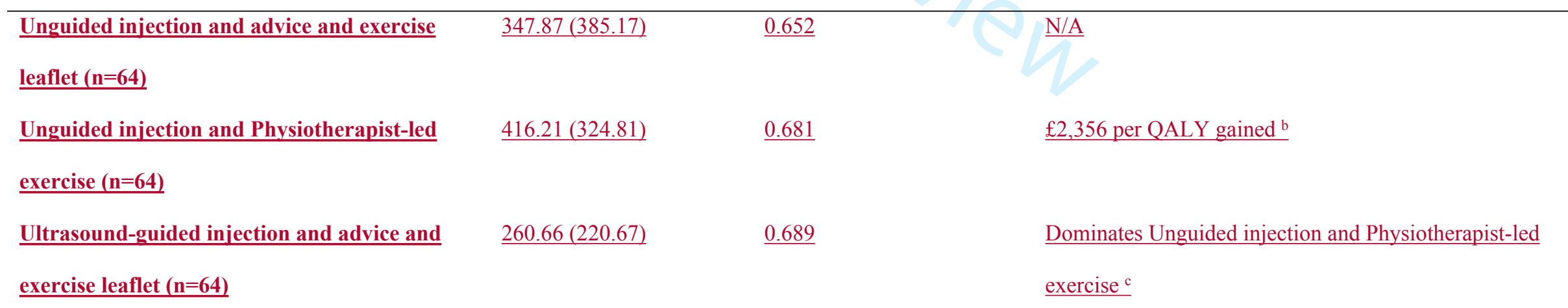




\section{Ultrasound-guided injection and}

\section{Physiotherapist-led exercise ( $\mathrm{n}=64)$}

*Cost and QALYs imputed ${ }^{\mathrm{a}}$ Adjusted for baseline utility ${ }^{\mathrm{b}}$ Unguided injection and Physiotherapist-led exercise versus Unguided injection and advice and exercise leaflet c Ultrasound-guided injection and advice and exercise leaflet versus Unguided injection and Physiotherapist-led exercise ${ }^{\mathrm{d}}$ Ultrasound-guided injection and Physiotherapistled exercise versus Ultrasound-guided injection and advice and exercise leaflet 
Supplementary Table 1: Unit cost of health care resource use

\begin{tabular}{cc}
\hline Health care resource & Unit cost \\
\hline Primary care contacts & 43 \\
GP at practice & 53.58 \\
GP at home & 10.30 \\
Nurse at practice & 23.30 \\
Nurse at home &
\end{tabular}

Secondary care contacts

$\begin{array}{ll}\text { Physiotherapist visits } & 34 \\ \text { Consultants } & 52 \\ \text { Specialists A\&E visits } & 62\end{array}$

Intervention $\operatorname{cost}^{\mathrm{a}}$

Physiotherapist-led exercise

Advice and exercise leaflet $\quad 1^{\mathrm{c}}$

$\begin{array}{ll}\text { Ultrasound-guided injection } & 65^{\mathrm{d}} \\ \text { Unguided injection } & 0^{\mathrm{e}}\end{array}$

Over the counter medication

Participant specific

Participant specific

Medical investigations/Interventions Participant specific

a These costs are what was actually used for the analysis ${ }^{\mathrm{b}}$ This value was multiplied by the number of times a participant saw a physiotherapist. Thus, the cost varied from participant to participant ${ }^{\mathrm{c}}$ This is the cost of the leaflet ${ }^{\mathrm{d}}$ Includes cost of the ultrasound procedure ${ }^{\mathrm{e}}$ Assumed to be zero since all patients had a similar injection and the only difference between the ultrasound-guided and unguided injection was the cost of the ultrasound. 
Supplementary Table 2: Mean (SD) NHS resource use per patient (complete cases)

\begin{tabular}{|c|c|c|c|c|c|c|}
\hline \multicolumn{4}{|c|}{ Injection intervention group } & \multicolumn{3}{|c|}{$\begin{array}{l}\text { Exercise intervention group } \\
\end{array}$} \\
\hline & US-guided ( $(n=95)$ & $\begin{array}{c}\text { Unguided } \\
(\mathrm{n}=99)\end{array}$ & $\begin{array}{l}\text { Difference } \\
\text { (Confidence } \\
\text { Interval) }\end{array}$ & $\begin{array}{c}\text { Physiotherapist-led } \\
(\mathrm{n}=95)\end{array}$ & $\begin{array}{l}\text { Advice and Exercise } \\
\text { Leaflet }(\mathrm{n}=99)\end{array}$ & $\begin{array}{l}\text { Difference } \\
\text { (Confidence } \\
\text { Interval) }\end{array}$ \\
\hline \multicolumn{7}{|c|}{ PRIMARY CARE CONTACTS } \\
\hline GP (surgery) & $0.80(1.51)$ & $0.90(2.03)$ & $\begin{array}{c}-0.10 \\
(-0.62,0.38)\end{array}$ & $0.57(1.41)$ & $1.12(2.07)$ & $\begin{array}{c}-\mathbf{0 . 5 5} \\
(-1.07,-0.07)\end{array}$ \\
\hline GP (home visit) & $0.01(0.10)$ & 0 & $\begin{array}{c}0.01 \\
(0.00,0.04)\end{array}$ & $0.01(0.10)$ & 0 & $\begin{array}{c}0.01 \\
(0.00,0.04)\end{array}$ \\
\hline Nurse visits (surgery) & $0.14(0.96)$ & $0.05(0.36)$ & $\begin{array}{c}0.09 \\
(-0.08,0.35)\end{array}$ & $0.11(0.84)$ & $0.08(0.58)$ & $\begin{array}{c}0.03 \\
(-0.14,0.28)\end{array}$ \\
\hline Nurse (home visit) & 0 & 0 & 0 & 0 & 0 & 0 \\
\hline $\begin{array}{r}\text { Other professionals } \\
\text { attached to GP practice } \\
\text { (surgerv) }\end{array}$ & $0.35(0.91)$ & $0.48(1.31)$ & $\begin{array}{c}-0.13 \\
(-0.46,0.17)\end{array}$ & $0.52(1.22)$ & $0.32(1.04)$ & $\begin{array}{c}0.20 \\
(-0.13,0.50)\end{array}$ \\
\hline $\begin{array}{r}{ }^{a} \text { Other professionals } \\
\text { attached to GP practice } \\
\text { (home) }\end{array}$ & $0.04(0.29)$ & $0.08(0.80)$ & $\begin{array}{c}-0.04 \\
(-0.26,0.08)\end{array}$ & $0.13(0.87)$ & 0 & $\begin{array}{c}0.13 \\
(0.02,0.38)\end{array}$ \\
\hline \multicolumn{7}{|c|}{ SECONDARY CARE CONTACTS } \\
\hline Consultant & $0.46(1.04)$ & $0.75(1.48)$ & $\begin{array}{c}-0.29 \\
(-0.64,0.08)\end{array}$ & $0.60(1.27)$ & $0.62(1.31)$ & $\begin{array}{c}-0.02 \\
(-0.40,0.35)\end{array}$ \\
\hline Physiotherapist ${ }^{\text {b }}$ & $2.79(3.96)$ & $2.45(3.78)$ & $\begin{array}{c}0.34 \\
(-0.67,1.47)\end{array}$ & $4.87(4.21)$ & $0.45(1.66)$ & $\begin{array}{c}4.42 \\
(3.51,5.35)\end{array}$ \\
\hline Specialist A\&E & 0 & $0.06(0.42)$ & $\begin{array}{c}-0.06 \\
(-0.19,-0.01)\end{array}$ & $0.04(0.41)$ & $0.02(0.14)$ & $\begin{array}{c}0.02 \\
(-0.04,0.12)\end{array}$ \\
\hline Hospital Nurse & 0 & $0.08(0.34)$ & $\begin{array}{c}-0.08 \\
(-0.17,-0.03)\end{array}$ & $0.02(0.14)$ & $0.06(0.31)$ & $\begin{array}{c}0.04 \\
(-0.12,0.02)\end{array}$ \\
\hline Acupuncturist & 0 & $0.01(0.10)$ & $\begin{array}{c}-0.01 \\
(-0.03,0)\end{array}$ & 0 & $0.01(0.10)$ & $\begin{array}{c}-0.01 \\
(-0.03,0)\end{array}$ \\
\hline c Other professionals & $0.06(0.35)$ & $0.06(0.31)$ & $\begin{array}{c}0 \\
(-0.08,0.10)\end{array}$ & $0.04(0.32)$ & $0.08(0.34)$ & $\begin{array}{c}-0.04 \\
(-0.13,0.07)\end{array}$ \\
\hline Repeat Injections & $0.26(0.72)$ & $0.37(0.86)$ & $\begin{array}{c}-0.11 \\
(-0.28,0.07)\end{array}$ & $0.24(0.77)$ & $0.39(0.82)$ & $\begin{array}{c}-0.15 \\
(-0.30,0.05)\end{array}$ \\
\hline
\end{tabular}

${ }^{\mathrm{a}}$ This includes contacts with professionals such as work nurse sister and consultant ${ }^{\mathrm{b}}$ Physiotherapy visits that were part of the intervention have been included here ${ }^{\mathrm{c}}$ Includes contacts with professionals such as work nurse sister, sports therapist, massage masseur and kinesiologist 
Supplementary Table 3: Mean (SD) prescribed drugs and medical investigations per patient over 12 months (complete cases)

\begin{tabular}{|c|c|c|c|c|c|c|}
\hline \multicolumn{4}{|c|}{ Injection intervention group } & \multicolumn{3}{|c|}{ Exercise intervention group } \\
\hline & US-guided (n=95) & Unguided (n=99) & $\begin{array}{c}\text { Difference } \\
\text { (Confidence } \\
\text { Interval) }\end{array}$ & $\begin{array}{l}\text { Physiotherapist-led } \\
\qquad(\mathrm{n}=95)\end{array}$ & $\begin{array}{l}\text { Advice and Exercise } \\
\text { Leaflet }(n=99)\end{array}$ & $\begin{array}{c}\text { Difference } \\
\text { (Confidence } \\
\text { Interval) }\end{array}$ \\
\hline \multicolumn{7}{|c|}{ PRESCRIBED DRUGS } \\
\hline Paracetamol & $0.38(1.45)$ & $0.52(2.00)$ & $\begin{array}{c}-0.14 \\
(-0.62,0.32)\end{array}$ & $0.37(1.39)$ & $0.53(2.04)$ & $\begin{array}{c}-0.16 \\
(-0.72,0.27)\end{array}$ \\
\hline Ibuprofen & $0.31(1.38)$ & $0.75(2.89)$ & $\begin{array}{c}-0.44 \\
(-1.14,0.08)\end{array}$ & $0.28(1.29)$ & $0.78(2.92)$ & $\begin{array}{c}-0.5 \\
(-1.24,0.06)\end{array}$ \\
\hline Co-codamol & $0.77(2.11)$ & $1.19(2.71)$ & $\begin{array}{c}-0.42 \\
(-1.13,0.24)\end{array}$ & $1(2.46)$ & $0.97(2.43)$ & $\begin{array}{c}0.03 \\
(-0.64,0.77)\end{array}$ \\
\hline Diclofenac & $0.04(0.25)$ & $0.09(0.81)$ & $\begin{array}{c}-0.05 \\
(-0.28,0.06)\end{array}$ & $0.01(0.10)$ & $0.12(0.84)$ & $\begin{array}{c}-0.11 \\
(-0.34,-0.001) \\
\end{array}$ \\
\hline \multicolumn{7}{|c|}{ MEDICAL INVESTIGATIONS } \\
\hline Blood test & $0.09(0.41)$ & $0.26(0.79)$ & $\begin{array}{c}-0.17 \\
(-0.04,-0.03)\end{array}$ & $0.05(0.22)$ & $0.30(0.85)$ & $\begin{array}{c}-0.25 \\
(-0.46,-0.10)\end{array}$ \\
\hline X-ray & $0.13(0.39)$ & $0.29(0.72)$ & $\begin{array}{c}-0.16 \\
(-0.33,-0.16)\end{array}$ & $0.16(0.55)$ & $0.26(0.62)$ & $\begin{array}{c}-0.10 \\
(-0.25,0.06)\end{array}$ \\
\hline Ultrasound & $0.11(0.35)$ & $0.33(0.73)$ & $\begin{array}{c}-0.22 \\
(0.39,-0.06)\end{array}$ & $0.17(0.54)$ & $0.28(0.62)$ & $\begin{array}{c}-0.11 \\
(-0.28,0.04)\end{array}$ \\
\hline MRI scan & $0.01(0.10)$ & $0.04(0.24)$ & $\begin{array}{c}-0.03 \\
(-0.10,0.01)\end{array}$ & $0.01(0.10)$ & $0.04(0.24)$ & $\begin{array}{c}-0.03 \\
(-0.10,0.01)\end{array}$ \\
\hline CT scan & 0 & $0.03(0.22)$ & $\begin{array}{c}-0.03 \\
(-0.10,0)\end{array}$ & 0 & $0.03(0.22)$ & $\begin{array}{c}-\mathbf{0 . 0 3} \\
(-\mathbf{0 . 1 0}, 0)\end{array}$ \\
\hline
\end{tabular}


Supplementary Table 4: Mean (SD) private resource use per patient over 12 months (complete cases)

\begin{tabular}{|c|c|c|c|c|c|c|}
\hline \multicolumn{4}{|c|}{ Injection intervention group } & \multicolumn{3}{|c|}{ Exercise intervention group } \\
\hline & US-guided (n=95) & $\begin{array}{c}\text { Unguided } \\
(\mathbf{n}=99)\end{array}$ & $\begin{array}{c}\text { Difference } \\
\text { (Confidence } \\
\text { Interval) } \\
\end{array}$ & $\begin{array}{l}\text { Physiotherapist-led } \\
(\mathrm{n}=95)\end{array}$ & $\begin{array}{l}\text { Advice and Exercise } \\
\text { Leaflet }(\mathbf{n}=99)\end{array}$ & $\begin{array}{c}\text { Difference } \\
\text { (Confidence } \\
\text { Interval) } \\
\end{array}$ \\
\hline Consultant & $0.12(0.63)$ & $0.05(0.36)$ & $\begin{array}{c}0.07 \\
(-\mathbf{0 . 0 6}, 0.24)\end{array}$ & $0.03(0.33)$ & $0.13(0.65)$ & $\begin{array}{c}-0.1 \\
(-0.26,0.03)\end{array}$ \\
\hline Physiotherapist & $0.11(0.83)$ & $0.06(0.42)$ & $\begin{array}{c}0.05 \\
(-1.1,0.28)\end{array}$ & $0.07(0.44)$ & $0.09(0.81)$ & $\begin{array}{c}-0.02 \\
(-0.26,0.13)\end{array}$ \\
\hline Specialist & $0.02(0.21)$ & 0 & $\begin{array}{c}0.02 \\
(0,0.08)\end{array}$ & 0 & $0.02(0.20)$ & $\begin{array}{c}-0.02 \\
(-0.10,0)\end{array}$ \\
\hline Hospital Nurse & $0.01(0.10)$ & $0.01(0.10)$ & $\begin{array}{c}0 \\
(-0.02,0.03)\end{array}$ & $0.01(0.10)$ & $0.01(0.10)$ & $\begin{array}{c}0 \\
(-0.02,0.04)\end{array}$ \\
\hline Acupuncturist & $0.01(0.10)$ & 0 & $\begin{array}{c}0.01 \\
(0,0.04)\end{array}$ & 0 & $0.01(0.10)$ & $\begin{array}{c}-0.01 \\
(-0.04,0)\end{array}$ \\
\hline Osteopath & $0.07(0.72)$ & $0.06(0.60)$ & $\begin{array}{c}0.01 \\
(-0.13,0.27)\end{array}$ & 0 & $0.13(0.92)$ & $\begin{array}{c}-0.13 \\
(-0.35,0)\end{array}$ \\
\hline Other professionals & $0.08(0.58)$ & $0.06(0.45)$ & $\begin{array}{c}0.02 \\
(-0.12,0.17)\end{array}$ & $0.04(0.41)$ & $0.10(0.60)$ & $\begin{array}{c}-0.06 \\
(-0.24,0.08)\end{array}$ \\
\hline $\begin{array}{l}\text { Over the counter } \\
\text { medication }\end{array}$ & $2.86(5.52)$ & $6.22(22.15)$ & $\begin{array}{c}-3.36 \\
(-9.61,-0.09)\end{array}$ & $5.24(21.60)$ & $3.94(8.78)$ & $\begin{array}{c}1.30 \\
(-2.08,7.57)\end{array}$ \\
\hline Repeat Injection & $0.01(0.11)$ & 0 & $\begin{array}{c}0.01 \\
(0,0.04) \\
\end{array}$ & & $0.01(0.10)$ & $\begin{array}{c}-0.01 \\
(-0.04,0)\end{array}$ \\
\hline & & & K RELATED & MS & & \\
\hline $\begin{array}{l}\text { Time off work due to } \\
\text { shoulder problems } \\
\text { (mean days) }\end{array}$ & $3.49(14.63)$ & $8.70(44.50)$ & $\begin{array}{c}-1.68 \\
(-13.44,6.33)\end{array}$ & $5.29(24.00)$ & $6.97(40.57)$ & $\begin{array}{c}-5.21 \\
(-16.73,1.93)\end{array}$ \\
\hline
\end{tabular}


Supplementary Table 5: Cost-utility analysis 'at the margins' approach (with EQ-5D-5L tariff)

\begin{tabular}{|c|c|c|c|c|c|c|}
\hline & \multicolumn{2}{|c|}{ Injection intervention group } & & \multicolumn{3}{|c|}{ Exercise intervention group } \\
\hline & $\begin{array}{l}\text { US-guided } \\
(n=128)\end{array}$ & $\begin{array}{l}\text { Unguided } \\
(n=128)\end{array}$ & & $\begin{array}{c}\begin{array}{c}\text { Physiotherapist-led } \\
(\mathrm{n}=128)\end{array} \\
\end{array}$ & $\begin{array}{c}\text { Advice \& Exercise } \\
\text { Leaflet } \\
(\mathbf{n}=128)\end{array}$ & \\
\hline & Mean & Mean & $\begin{array}{l}\text { Mean difference } \\
\text { (Confidence } \\
\text { Interval) }\end{array}$ & Mean & Mean & $\begin{array}{l}\text { Mean difference } \\
\text { (Confidence } \\
\text { Interval) }\end{array}$ \\
\hline \multicolumn{7}{|c|}{ HEALTHCARE PERSPECTIVE } \\
\hline Cost & $£ 428.01$ & $£ 412.12$ & $\begin{array}{c}£ 15.89 \\
(-59.36,109.86)\end{array}$ & $£ 498.06$ & $£ 342.07$ & $\begin{array}{c}£ 155.99 \\
(69.02,241.93)\end{array}$ \\
\hline QALYs ${ }^{a}$ & 0.697 & 0.667 & $\begin{array}{c}0.030 \\
(-0.009,0.070)\end{array}$ & 0.693 & 0.671 & $\begin{array}{c}0.023 \\
(-0.017,0.062)\end{array}$ \\
\hline ICER & $£ 529.7$ per QALY gained & & & $£ 6,782$ per QALY gained & & \\
\hline \multicolumn{7}{|c|}{ NHS PERSPECTIVE } \\
\hline Cost & $£ 398.25$ & $£ 382.04$ & $\begin{array}{c}£ 15.85 \\
(-6432.9022)\end{array}$ & $£ 476.02$ & $£ 304.27$ & $\begin{array}{c}£ 171.75 \\
(9844 \quad 251.88)\end{array}$ \\
\hline QALYs a & 0.697 & 0.667 & $\begin{array}{c}0.030 \\
(-0.009,0.070)\end{array}$ & 0.693 & 0.671 & $\begin{array}{c}0.023 \\
(-0.017,0.062)\end{array}$ \\
\hline ICER & $£ 528.3$ per QALY gained & & & $£ 7,467$ per QALY gained & & \\
\hline
\end{tabular}

${ }^{a}$ Adjusted for baseline utility 
Supplementary Table 6: Cost-utility analysis "within the table" analysis (With EQ-5D-5L tariff)

\begin{tabular}{|c|c|c|c|}
\hline & $\operatorname{Cost}(\mathfrak{f}) *$ & QALY*a & ICER \\
\hline & \multicolumn{3}{|c|}{ Health care perspective } \\
\hline Unguided injection and advice and exercise & $385.31(397.70)$ & 0.652 & N/A \\
\hline \multicolumn{4}{|l|}{ leaflet $(n=64)$} \\
\hline Unguided injection and Physiotherapist-led & $438.92(332.49)$ & 0.681 & $£ 1,848$ per QALY gained ${ }^{b}$ \\
\hline \multicolumn{4}{|l|}{ exercise $(n=64)$} \\
\hline Ultrasound-guided injection and advice and & $298.82(281.99)$ & 0.689 & Dominates Unguided injection and Physiotherapist-led \\
\hline exercise leaflet $(n=64)$ & & & exercise $^{c}$ \\
\hline Ultrasound-guided injection and & $557.20(310.90)$ & 0.705 & $£ 16,148$ per QALY gained ${ }^{d}$ \\
\hline Physiotherapist-led exercise $(n=64)$ & & & \\
\hline
\end{tabular}

NHS perspective

\begin{tabular}{|c|c|c|c|}
\hline $\begin{array}{l}\text { Unguided injection and advice and exercise } \\
\text { leaflet }(n=64)\end{array}$ & $347.87(385.17)$ & 0.652 & N/A \\
\hline $\begin{array}{l}\text { Unguided injection and Physiotherapist-led } \\
\text { exercise }(n=64)\end{array}$ & $416.21(324.81)$ & 0.681 & $£ 2,356$ per QALY gained $^{\mathrm{b}}$ \\
\hline $\begin{array}{l}\text { Ultrasound-guided injection and advice and } \\
\text { exercise leaflet }(n=64)\end{array}$ & $260.66(220.67)$ & 0.689 & $\begin{array}{l}\text { Dominates Unguided injection and Physiotherapist-led } \\
\text { exercise }^{c}\end{array}$ \\
\hline
\end{tabular}




\section{Physiotherapist-led exercise $(n=64)$}

*Cost and QALYs imputed ${ }^{\mathrm{a}}$ Adjusted for baseline utility ${ }^{\mathrm{b}}$ Unguided injection and Physiotherapist-led exercise versus Unguided injection and advice and exercise leaflet

${ }^{c}$ Ultrasound-guided injection and advice and exercise leaflet versus Unguided injection and Physiotherapist-led exercise ${ }^{\mathrm{d}}$ Ultrasound-guided injection and Physiotherapistled exercise versus Ultrasound-guided injection and advice and exercise leaflet 